\title{
1 A web-based software tool for participatory optimization of conservation practices in
}

2 watersheds

4 Meghna Babbar-Sebens ${ }^{1,3}$, Snehasis Mukhopadhyay ${ }^{2}$, Vidya Bhushan Singh ${ }^{2}$, Adriana Debora 5 Piemonti ${ }^{1}$

${ }^{1}$ School of Civil and Construction Engineering, Oregon State University, Corvallis, OR 97331

$82^{2}$ Department of Computer \& Information Science, Indiana University - Purdue University 9 Indianapolis, Indianapolis, IN 46202

${ }^{3}$ Corresponding author, meghna@oregonstate.edu, 1-541-737-8536

\section{Highlights:}

1. A participatory design tool that uses interactive and human-guided approaches to simulation-optimization has been developed for planning of conservation practices

2. Users can be engaged to view and evaluate designs based on quantifiable and unquantifiable criteria

3. The software is web-based and can be used for engagement with individual users or multiple users

ABSTRACT: WRESTORE (Watershed Restoration Using Spatio-Temporal Optimization of Resources) is a web-based, participatory planning tool that can be used to engage with watershed stakeholder communities, and involve them in using science-based, human-guided, interactive simulation-optimization methods for designing potential conservation practices on their

24 landscape. The underlying optimization algorithms, process simulation models, and interfaces

25 allow users to not only spatially optimize the locations and types of new conservation practices

26 based on quantifiable goals estimated by the dynamic simulation models, but also to include their

27 personal subjective and/or unquantifiable criteria in the location and design of these practices. In this paper, we describe the software, interfaces, and architecture of WRESTORE, provide scenarios for implementing the WRESTORE tool in a watershed community's planning process, and discuss considerations for future developments.

This is the author's manuscript of the article published in final edited form as:

Babbar-Sebens, M., Mukhopadhyay, S., Singh, V. B., \& Piemonti, A. D. (2015). A web-based software tool for participatory optimization of conservation practices in watersheds. Environmental Modelling \& Software, 69, 111127. http://doi.org/10.1016/j.envsoft.2015.03.011 
32 Keywords: interactive optimization, participatory design, conservation practices, web-based, 33 subjective criteria, watershed planning and management.

\section{SOFTWARE AVAILABILITY}

36 Name of software: WRESTORE (Watershed REstoration using Spatio-Temporal Optimization

37 of Resources)

Developers: Vidya Bhushan Singh, Meghna Babbar-Sebens, Adriana Debora Piemonti, and

39 Snehasis Mukhopadhyay

40 First available year: 2014

41 Software requirements: Web-browser

42 Programming language: Java

43 Language: English

44 Minimum hardware requirements: Intel Pentium II, $200 \mathrm{MHz}, 128 \mathrm{MB}$ RAM

45 Contact person: Meghna Babbar-Sebens (Corresponding author)

46 URL: http://wrestore.iupui.edu/

\section{INTRODUCTION}

49 Recently, there has been an increased effort to help mitigate the effects of increased climate change induced flooding by restoring degraded upland and downstream storage capacities of

51 watersheds via conservation practices. For example, Hey et al. (2004) reported that the 80-day

52 Mississippi River flood in 1993 - which generated 48 billion cubic meters (or, 39 million acre-

53 feet) of floodwaters at St Louis, MO - could have been contained within the 49 billion cubic 54 meters (or, 40 million acre-feet) storage that could have been provided by adding storage 55 capacities of the drained wetlands to the existing levees and existing wetlands. Lemke and 56 Richmond (2009) and Babbar-Sebens et al. (2013) have also suggested that re-naturalization of 57 the hydrologic cycle with best management practices (or, conservation practices) on the 58 landscape can solve both water quantity and water quality problems in mixed land use watersheds. However, design of a system of conservation practices for upland storage is a complex process because there can be a large number of alternative sites, scales, and mitigation

61 methods, and because - with multiple stakeholders - there can be multiple criteria and 62 constraints for selection among alternatives. Additionally, achieving the desired level of 
restoration in a watershed will depend not only on the diverse costs and benefits of modifying the landscape but also on whether the landowners and other stakeholders will find prescribed practices acceptable when they are constrained by their subjective perceptions, uncertainty in human behavior, and local field-scale conditions (Wilcove, 2004). Therefore, successful restoration of hydrology requires obtaining a thorough understanding of the people and ecological processes that are unique to the watershed system, and then using this understanding in the design of appropriate management alternatives for restoring/creating upland storage systems.

Designing or generating alternatives is an integral part of problem-solving and decision making processes. In commonly used models (and their adaptations) of decision-making processes, such as those proposed by Mintzberg et al. (1976) and Simon (1977), the design of alternatives usually occurs in the second phase of a three phase process that includes - (1) problem identification and definition phase, (2) problem development and alternatives generation phase, and (3) negotiation and selection phase. The first phase involves interaction with stakeholders and experts to identify, structure, and define the problem. For example, for the restoration problem, this would involve developing a conceptual model of the combined human-physical system, and quantitatively defining the various objectives and constraints of the restoration project based on projects costs, economic benefits, environmental benefits, and stakeholder values and preferences. Conducting interviews with stakeholders and constructing quantitative economic valuation of the various ecosystem services provided by the upland storage systems would be an integral part of this phase. The second phase involves use of various computational tools, such as, simulation models and search/optimization algorithms. These models and algorithms along with the parameters of the search/optimization algorithm, and quantitative representations of the problem objectives and constraints defined in Phase 1, are then used to generate optimized sets of alternatives (or, scenarios of solutions) that would satisfy or outperform the problem objectives. When multiple conflicting objectives exist in a natural resource planning and management problem, a non-dominated set of alternatives are generated by the optimization algorithms, which is also called the Pareto-optimal set or a tradeoff curve. This phase is computationally intensive, and generally assumes that multiple stakeholder values and preferences obtained in Phase 1 can be quantified and reliably used to search for alternatives 
and to generate a search outcome for Phase 3. Once, the search has ended in Phase 2, the alternatives are then presented to the stakeholders in Phase 3 for decision making and selecting a final alternative for implementation. Many multi-criteria decision aid techniques exist in the literature (Haimes and Hall, 1974; Soncini-Sessa et al. 2007; Assaf et al. 2008; Castelletti and Soncini-Sessa (2006, 2007)), which can be used to include stakeholder feedback to select the “final” alternative in Phase 3 from a set of optimized non-dominated optimal alternatives, based

100

101

102

103

104

105

106

107

108

109

110

111

112

113

114

115

116

117

118

119

120

121

122

123

124 on multiple quantitative and qualitative criteria. However, by the time the stakeholders reach Phase 3 for decision making it is typically assumed that the search/optimization process in Phase 2 has used an accurate or close to accurate representation of the stakeholder criteria, and, therefore, alternatives optimized for these quantitative representations will be "optimal" solutions to the problem. This is, however, not true since in real-world watershed problems there can also be local knowledge, non-quantifiable beliefs and values, and incomplete/unstated preferences of the stakeholders that may not be captured in simulation-optimization models (Andradóttir, 1998; Fu, 1994, 2002; Gosavi, 2003; Law and Kelton, 2000). This can lead to stakeholders' dissatisfaction with the optimized alternatives and poor adoption of prescribed alternatives (Soncini-Sessa et al. 2007). In summary, though many methods in the literature have been developed for incorporating active stakeholder involvement in Phases 1 and 3, active involvement of stakeholders has been limited in the search and design process (i.e., Phase 2).

With the current trend of water resources planning and management approaches becoming more “bottom-up” or participatory (Assaf et al. 2008; Voinov and Bousquet, 2010; McIntosh et al., 2011; Döll et al. 2013; Hamilton et al., 2015), where stakeholders are involved in all stages of modeling and planning, the need for better understanding of people-related processes in design of alternatives has become ever more crucial. Involving stakeholders in the multiple steps of the decision making process, including the alternatives generation phase (i.e. Phase 2), can yield multiple benefits (Bierle, 1999; Daniels and Walker, 2001; Selin et al., 2007). For example, stakeholder involvement (a) gives individuals a sense of ownership in the decision process by allowing them to directly influence the problem-solving process, (b) provides a platform for open and honest expression of stakeholder views, and (c) improves the legitimacy of the planning and management process, while also conveying the complexities and uncertainties associated with this process to the public. With ongoing developments in Web technologies, the internet has the 
125

126

127

128

129

130

131

132

133

134

135

136

potential to be a robust medium for supporting participation of and communication between stakeholders in natural resources management (Esty, 2004; Rinner et al., 2008; Kelly et al., 2012). Kelly et al. (2012) reports that most of the current research in using the Web in natural resources management has been focused on (a) information delivery to the public by government agencies, with the ability for public to comment on on-line documents (e.g., Beckley et al., 2006; Conrad and Hilchey, 2011), (b) interactive social-web tools for harnessing (or “crowd-sourcing”) feedbacks from large groups of individuals via on-line dialogues and discussions (e.g., Kangas and Store, 2003; O’Reilly, 2007; Hudson-Smith et al., 2009), and (c) development of mapping and other spatial decision support tools for effectively communicating spatial data to support decision making (e.g., Kearns et al., 2003; Sheppard and Meitner, 2005; Brown and Reed, 2009; Brown and Weber, 2011). It is worthwhile to note that none of the existing technologies and software cited in these studies provide a truly human-computer collaborative design environment where stakeholders can participate in design experiments to visualize alternatives and provide feedbacks on both the design features and acceptability of system-generated alternatives, and in return have that feedback used to generate new community-preferred alternatives of natural resources management plans.

In a 1985 seminal paper, Fisher (Fisher, 1985) motivated a discussion on optimization/search algorithms that were interactive and allowed humans to be a part of the search process, especially for problems where human thought processes would provide "superior" advantage to the “algorithmic thinking” employed by a computer - for example, processes related to visual perception, strategic thinking, and the ability to learn. According to his discussions, incorporating human interaction within the optimization algorithms could - (a) facilitate model specification and revisions, (b) help cope with problem aspects that are difficult to quantify, and (c) assist in the solution process. A human-computer collaborative decision support framework that uses such a search process would allow stakeholders real-time access to influence the search process of the optimization algorithm by influencing the definition of objectives and constraints, the characterization of alternatives, the simulation models, and algorithm parameters. This not only allows a more flexible and transparent framework for including stakeholders preferences and subjective knowledge to construct meaningful, better performing, and desirable (from the perspective of both humans and quantitative evaluation objective functions) alternatives; it also 
creates a venue for improving the cognitive learning process of the interacting human (BabbarSebens and Minsker, 2012). Also known as human-guided search (Klau et al., 2009), the interactive search/optimization process has been explored in applications such as space shuttle scheduling (Chien et al. 1999), vehicle routing (Waters 1984), face image generation (Takagi, 2001), and constraint-based graph drawing (do Nascimento and Eades, 2002). In recent work by Babbar-Sebens and Minsker (2012), heuristic Genetic Algorithms were examined as interactive optimization methods for solving a ground water monitoring problem. In their research, the authors proposed an innovative algorithm, Interactive Genetic Algorithm with Mixed Initiative Interaction (IGAMII), which examined the effect of including a single decision maker in the optimization algorithm's loops (i.e. human-in-the-loop) to guide the search process. The main aim of the interactive optimization process was to enable the user to assist the optimization algorithm find solutions in the "region of desirable solutions," which could be more optimal from the user's non-quantifiable perspective than the solutions on the Pareto front found via a typical non-interactive search and based on only the quantified representative objectives. It is this region of desirable solutions that are of most interest to the decision maker since their subjective evaluation by the user will be complemented by their performance in the quantitative evaluations. Effects of various human factors, such as human fatigue, non-stationarity in preferences, and the cognitive learning process of the human decision maker on the search process of the interactive genetic algorithm were also addressed in their research.

In this paper, we present the development of a new, web-based, interactive optimization tool, Watershed REstoration using Spatio-Temporal Optimization of Resources (WRESTORE), which is based on the IGAMII algorithm and provides a participatory environment for generating individual and community-preferred alternatives of conservation practices in watersheds. Unlike the original desktop-based IGAMII algorithm and other participatory desktop-based planning tools (e.g., WEAP by Yates et al., 2005a, 2005b; Catchment Simulation Shell by Argent and Grayson, 2003), WRESTORE uses Web 2.0 technologies to reach out to larger stakeholder communities for participatory planning efforts and in crowdsourcing the design of potential conservation practices in a watershed. In this manner, the tool can be used to engage multiple, diverse watershed stakeholders and landowners via the internet, thereby improving opportunities for outreach and collaborations. Multiple visualization interfaces, computational simulation and 
optimization models, and user modeling, and engagement techniques are part of the WRESTORE methodology to support a human-centered design approach. Users are able to (a) design multiple types of conservation practices in their sub-basins and at the entire watershed scale, (b) examine impacts and limitations of their decisions on their neighboring catchments and on the entire watershed, (c) compare alternatives via a cost-benefit analysis, (d) vote on their "favorite" designs based on their preferences and constraints, and (e) propose their "favorite" alternatives to policy makers and other stakeholders. This human-centered design approach, which is reinforced by use of internet technologies, has the potential to enable policy makers to connect to a larger community of stakeholders and directly engage them in environmental stewardship efforts. The use of web-based interaction technologies also enable an improved understanding of how users explore alternatives that interest them, learn from making choices in a safe simulated environment, and change their perceptions of alternatives. This issue is also especially important in the context of agricultural landowners whose mental maps, perceptions, behaviors and attitudes affect their understanding of their environment and their intrinsic motivation to adapt to the changing environment. For example, McCown (2002) insisted that a paradigm shift is needed in the implementation of decision support systems, specifically a "shift in emphasis from 'design' to 'learning,' without abandoning design. Users must undergo an iterative learning and practice change process. The researchers must be prepared to be involved in, lend support to, and learn from this process-learn what the farmers are learning”. Moreover, the software and decision support tool developed is this research provides a framework for investigations on similar human-centered and web-based participatory design technologies in the future. While this paper only presents the software development and testing of the participatory design tool, multiple research investigations on the simulation models, algorithms, user-learning, etc. supported by WRESTORE have been (e.g., Babbar-Sebens and Minsker 2012; Babbar-Sebens et al, 2012; and Piemonti et al., 2013) and will be presented in separate research articles.

\section{WRESTORE SOFTWARE DESCRIPTION}

2.3. Representation of Conservation Practices in WRESTORE: Seven conservation practices are currently modeled in WRESTORE - Wetlands, Filter Strips, Grassed Waterways, 
Strip Cropping, Cover crops, Crop Rotation, and No-till tillage practice. The main goal of the WRESTORE tool is to assist stakeholders in identifying the most effective spatial distribution and design of conservation practices (or, best management practices (BMPs)) in the various subbasins of their watershed. Users have the ability to select one or more practices from the candidate practices being considered for a watershed, and the spatial design is based on decisions made by the underlying optimization algorithm for every practice in every sub-basin. For example, if a watershed has $N$ number of sub-basins where practices can be implemented, and if a user wants to consider all seven practices in the $N$ sub-basins, then WRESTORE's underlying optimization algorithm will assign values to decision variables representing these practices in the following manner (see Babbar-Sebens et al. (2013) and Piemonti et al. (2013) for more details):

(i) Strip cropping, crop rotation, no-till, cover crops, and grassed waterways: These five practices are all modeled as binary decisions, $\mathrm{x}_{i j}$, which can have a value of 1 (when the practice is proposed for implementation in a sub-basin) or 0 (when the practice is not implemented in a sub-basin). The sub-script $i$ is the designated ID of each of these five practices in WRESTORE and is used to identify the practice. The sub-script $j$ stands for every sub-basin where practices can be implemented, and it varies from 1 to $N$.

(ii) Filter strips: This practice is modeled as a real number decision variable $y_{i j}$, which is the width of the filter strip along a stream in the $j^{\text {th }}$ sub-basin. The sub-script $i$ is the designated ID of the filter strip practice in WRESTORE. The range of values between which a decision on filter strip widths can vary have to be determined before an experiment (e.g., minimum value $=0 \mathrm{~m}$ and maximum value $=50$ meters).

(iii) Wetlands: Two real-valued decision variables, $y_{i j}$, for each sub-basin are used to identify the design of wetlands across sub-basins - one on the maximum wetland area (WET_MXSA) and one on the fraction of sub-basin area that drains into the wetland (WET_FR). Subscript $i$ is the designated practice ID of the two wetland decision variables WET_MXSA and WET_FR in WRESTORE, and subscript $j$ is the ID of the sub-basin respectively. The minimum and maximum values of these variables for every sub-basin need to be provided to WRESTORE, and, if not easily available for a watershed, can be determined using a GIS methodology proposed by Babbar-Sebens et al. (2013). 
250 WRESTORE's underlying optimization algorithm (discussed in detail in sections below) will generate a large number of map scenarios or map alternatives, where each alternative has a 252 unique spatial combination of the decision variables related to the practices (e.g., Figure 1 shows 253 an example of Decision Alternatives by using icons and colors on a map to indicate values of individual sub-basin decision variables for each practice). However, to simulate effectiveness of all of these alternatives, decision variables are mapped into hydrologic and environmental variables in the watershed model chosen by a community to simulate conservation practices in 257 the specific watershed (as shown in the Process Simulation box in Figure 1). Currently, we use the Soil and Water Assessment Tool (SWAT (Arnold et al., 2001, 2005)) to simulate individual practices in WRESTORE. While details on how each practice is simulated in SWAT can be found elsewhere (e.g., Bracmort et al. (2006), Arabi et al. (2007), Piemonti et al. (2013), and Rabotyagov et al. (2013)), here we only provide a brief summary on how the decisions would be mapped into specific input variables for the SWAT model based on our earlier study (Piemonti et al. (2013)):

(i) Strip Cropping: This practice increases the surface roughness, and reduces surface

(ii) Crop Rotation: This practice improves soil quality, creating a balance of nutrients in

(iii) Cover Crops: This practice helps in improving soil moisture content, minimizing soil compaction, preventing erosion, and increasing soil organic matter. This practice is generally implemented at the time when land is not being used for production 
(winter/spring). The SWAT model allows scheduling of more than one cover crop per year, once in the fall and once in spring. When a sub-basin has decision variable $x_{i j}=$ 1 for this practice, then the most common cover crop operations schedule for the watershed is used in the crop-related .mgt files of that sub-basin.

(iv) Filter Strips: This practice reduces suspended solids and associated contaminants in the runoff. It is generally implemented on the edges of channel segments. Based on the value of the decision variable $y_{i j}$ for this practice, the FILTERW (Filter width) variables in .mgt files of that sub-basin are replaced by the $y_{i j}$ value.

(v) Grassed Waterways: This practice reduces gully erosion, reduces flow velocity and increases sediment settlement (Arabi et al., 2007). Sub-basins with first-order streams are allowed to have this practice in WRESTORE. When such a sub-basin has decision variable $x_{i j}=1$ for this practice, the variable CH_COV (Channel cover factor) is modified in the .rte file of that sub-basin. See Piemonti et al. (2013) for details on how an appropriate value for this parameter can be determined.

(vi) No-Till: This practice increases the amount of organic matter and moisture in the soil, and also decreases erosion. When a sub-basin has decision variable $x_{i j}=1$ for this practice, the tillage operation in the operation schedule in the crop related .mgt files of the sub-basin is replaced by a no till operation commonly implemented in the watershed.

(vii) Wetlands: Wetlands reduce sediments in runoff, reduce peak flows in streams, reduce nutrient loads in runoff, and also provide habitat for wildlife. Wetlands are simulated in SWAT as water bodies at outlets of sub-basins, with a maximum of one wetland at every outlet. The SWAT variables wet fraction (WET_FR) and maximum wetland area (WET_MXSA) in the .pnd files of each sub-basin are replaced by the values of the related decision variable $y_{i j}$. See Babbar-Sebens et al. (2013) for details on how appropriate values for these parameters can be determined

Once the decision variables of an alternative have been mapped into appropriate input variables for the watershed model (e.g., the SWAT model in the current version of WRESTORE), the input files of the model are updated, and the process simulation model is then run for a specific period of simulation time. The output files generated by the model can next be used to estimate 
311 performance of the practices proposed in this alternative. Performance can be estimated for a 312 short time period or long time period, based on how long the simulation was run for. Currently 313 five types of performance measures are available in WRESTORE (see Figure 1), with the plan to 314 add more. The first one is called user rating that is provided by the user during the WRESTORE 315 experiment (described in Sections 2.2-2.5) and serves as a representation of the user's subjective criteria and preference for an alternative. The other four of these performance measures are used as quantitative Objective Functions (or, quantitative criteria) by the underlying optimization algorithm (described in sections below), and can be estimated for each sub-basin and also for the entire sub-basin from the physical state variables in model output files. Here we only provide a brief summary on how these performance measures are calculated based on our earlier study 321 (Piemonti et al. (2013)):

Cost-revenue function: This objective function considers the costs and revenues generated by the conservation practice over model time period T1-T2 (in years). It represents net present values (across all $N$ sub-basins) of all economic costs and revenues that the conservation practices would accrue for the landowner investing in this practice at a sub-basin $j$, and is given by:

$E C=\min \left[\sum_{j=1}^{N} N P V_{j}\right]$ where, $N P V_{j}$ (or Net Present Value of Economic Costs in US dollars at a sub-basin $j$ ) is calculated using,

$N P V_{j}=\sum_{i=1}^{B M P}\left[C I_{i} * A_{j, i}\right]+\sum_{t y=T 1}^{T 2}\left\{\sum_{i=1}^{B M P}\left[\left(O M_{i, t y}-\operatorname{Rin}_{i, t y}\right) * A_{j, i}\right]-P I_{t y}-S P_{t y}\right\} *$ $P W F_{t y}$

Where, $i$ is the specific conservation practices out of BMP number of practices, $C I_{i}$ is the cost of implementation in dollars per acre for each conservation practice, $A_{j, i}$ is the area in acres of the conservation practice $i$ in a sub-basin $j$, ty is the year that varies from $T 1$ to $T 2, O M_{i, t y}$ is the operation and maintenance cost in dollars per acre per each conservation practice $i$ in year ty, $\operatorname{Rin}_{i, t y}$ is the rent received by the conservation program in dollars per acre for those lands that are taken out of production for the conservation practice $i$ in year ty, $S P_{t y}$ is the savings in costs of crop productions in dollars of taking land out of production for conservation practice in year ty, $P I_{t y}$ represents the net profits, in dollars, obtained from increased productivity in year ty. PWF is the single payment present worth per year based on interest rate int and is 
given by Equation 3 below. Details on calculation of individual terms in Equation 2 can be obtained from Piemonti et al. (2013).

(ii) Peak flow reduction function: Peak flow reduction represents impact on flooding and is calculated based on the maximum difference between the peak flows of the calibrated baseline model without any new conservation practices and peak flows of the model that includes conservation practices proposed by an alternative found via the optimization algorithm. Equation (4) presents the equation for this objective function. The main goal of this function is to maximize the maximum peak flow reduction in the watershed across all sub-basins, or in other words minimize the negative of the maximum peak flow reduction.

$P F R=\min \left[-\max _{i, t}\left(\right.\right.$ peakflow $_{i, t, \text { baseline }}-$ peaflow $\left.\left._{i, t, \text { alternative }}\right)\right]$

where PFR is the peak flow reduction, $i$ is the sub-basin ID, $t$ is the day in modeled time period T1-T2 years, peakflowi,t,baseline are the baseline peak flows when no new conservation practice exists in the watershed, and peakflowi,t,alternative are the modeled peak flow when the alternative consisting of a specific combination of conservation practices exists in the watershed in sub-basin $i$, and time $t$. The peak flows in equation (4) can be determined from simulated daily flows at the outlet of every sub-basin (i.e., flowout $_{i, t, \text { case }}$ ) for any case (i.e. case = baseline or case = alternative) via equation (5) below:

(iii) Sediments reduction function: Sediments reduction objective function (SR) is calculated as per equation (6). This function represents the loss of fertile soil from the landscape, across all sub-basins $(N)$ and for the days in time period T1-T2 years. The main goal of this function is to maximize sediments reduction in all sub-basins, or, in other words, minimize the negative of sediments reduction in all sub-basins.

where $i$ is the sub-basin ID, $t$ is time in days (e.g., day 367), Sedout $t_{i, t, b a s e l i n e}$ is the sediments load at the outlet of sub-basins for the baseline calibrated model that does 
not have any new conservation practices, and Sedout $t_{i, t, a l t e r n a t i v e}$ is the sediments load at the outlet of sub-basins when the WRESTORE generated alternative with a specific spatial combination of conservation practices is simulated by the watershed model.

(iv) Nitrates reduction function: Nitrates reduction objective function (NR) is calculated as per equation (7). This function represents loss in nitrates via runoff, including those originating from the applied fertilizers, across all sub-basins $(N)$ and for the days in time period T1-T2 years. The main goal of this function is to maximize nitrates reduction in all sub-basins, or, in other words, minimize the negative of nitrates reduction in all sub-basins.

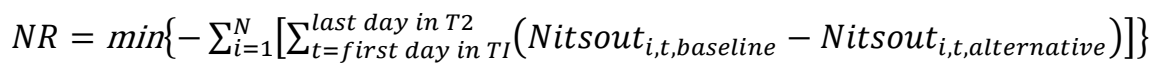

where $i$ is the sub-basin ID, $t$ is time in days (e.g., day 367), Nitsout $t_{i, t, b a s e l i n e}$ is the nitrates load at the outlet of sub-basins for the baseline calibrated model that does not

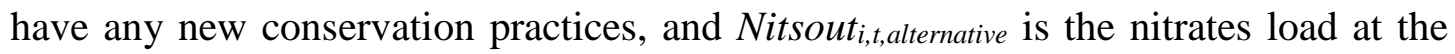
outlet of sub-basins when the WRESTORE generated alternative with a specific spatial combination of conservation practices is simulated by the watershed model.

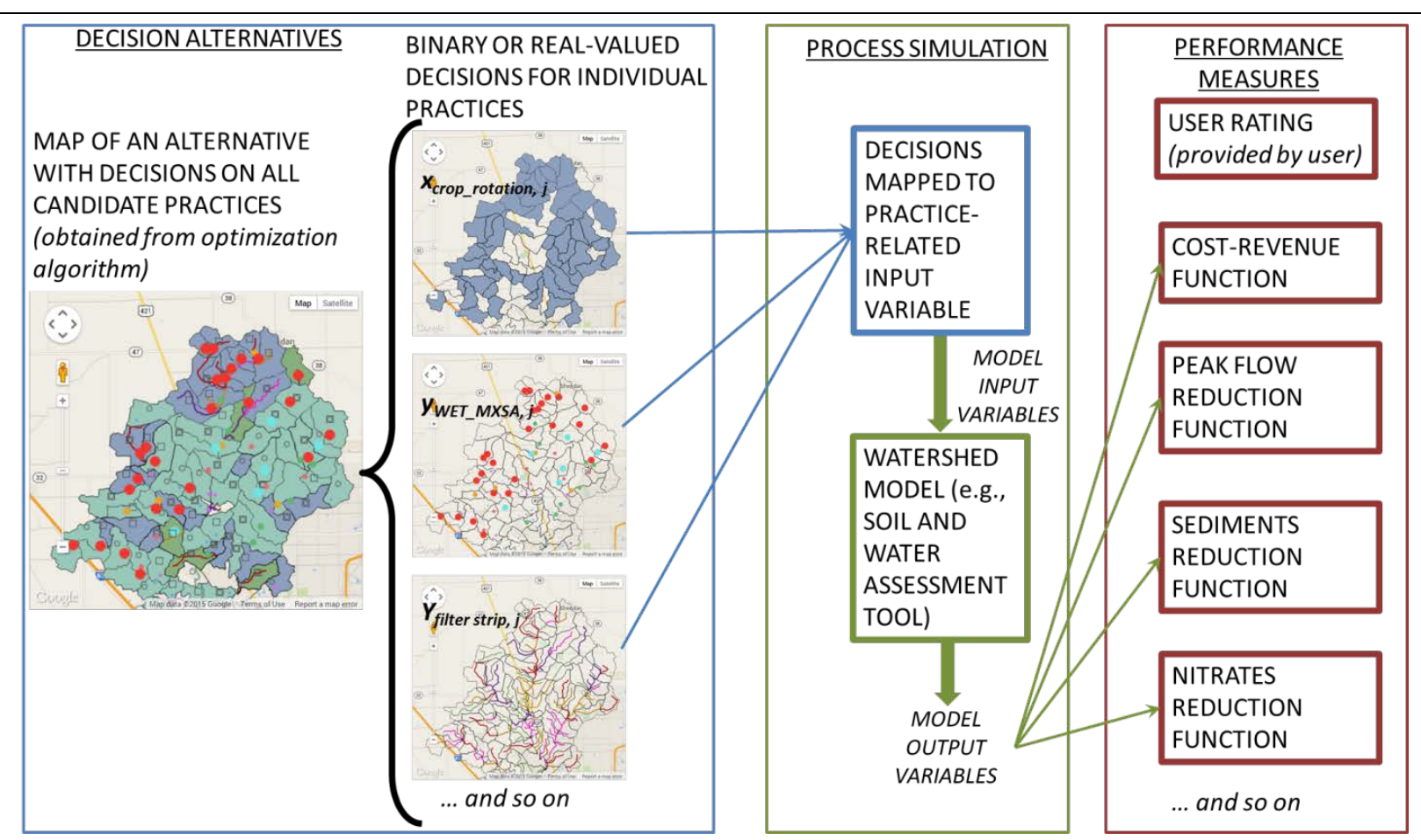

Figure 1. Conservation practices in WRESTORE- Decision Alternatives, Process Simulation, and Measures of Performance. 
2.2. Participatory Optimization Methodology: As mentioned above, the participatory optimization approach in web-based WRESTORE software is similar to the Interactive Genetic Algorithm with Mixed Initiative Interaction (IGAMII) algorithm proposed originally by BabbarSebens and Minsker (2012). We describe here a summary of the IGAMII algorithm, and the reader is advised to refer to their study for methodological details.

The IGAMII algorithm is a human-guided (or, human-centered) optimization algorithm that engages with human users/stakeholders in an iterative manner via visualization interfaces. In every iteration, which is called an interaction session, both the decision space of the alternatives (via maps) and the objective space of the alternatives (via graphs) are displayed to the user. The user evaluates multiple alternatives based on not only the quantitative objectives (i.e. mathematical functions of cost-benefit type goals) but also based on the user's local subjective criteria or qualitative knowledge not represented in the problem formulation. Once the user has evaluated the alternatives, she/he can provide her/his feedback on the quality of the alternative to the IGAMII's underlying optimization algorithm via a user rating or human rank determined on a Likert type psychometric scale (e.g. "good”, "average”, "bad”, etc.). The IGAMII's optimization algorithm uses this user rating as an additional user-driven objective function (in addition to economic and physical objectives discussed in Section 2.1) to identify new alternatives that are similar to or better than the alternatives liked by the user. The underlying optimization algorithm is critical to enabling the search of new alternatives, and though the IGAMII uses a multi-objective Genetic Algorithm called NSGA-II (Deb et al., 2002), WRESTORE is not restricted by the type of multi-objective optimization technique and has the capabilities to select from a variety of other search approaches (e.g., Decentralized Pursuit Learning Automata (Singh, 2013)).

Interaction sessions in IGAMII can be of three types (see Figure 2 that shows the sequence of sessions in an example experiment): introspection sessions, human-guided search (HS) sessions, and automated search sessions. An introspection session is used for improving the learning efficiency of the human user by enabling the user to re-examine previously viewed and rated alternatives that are stored in a case-based memory (Craw, 2003; Shi and Zhang, 2005), and re-assess her/his own thoughts, reasoning process, emotions, biases, consciousness, and user ratings of these previously assessed alternatives. For example, Figure 2 illustrates an IGAMII experiment in which five introspection sessions occurred at different times during the progress of 
the experiment. Each of the human-guided search (HS) sessions is an iteration of the underlying optimization technique (or, generation in the case when a Genetic Algorithm is used as the search method in IGAMII), where new alternatives created by the underlying optimization operators are shown to the user. In IGAMII, when human-guided search is conducted, a small population micro-genetic algorithm is used. Hence the number of alternatives shown in a typical HS session is typically equal to the population size of this micro-genetic algorithm. Every alternative (or, the genetic algorithm chromosome) is evaluated in its performance using a suite of mathematical objective functions and process simulation models (e.g., the SWAT model of a watershed); and then the values of these performance-based objective functions are displayed to the user, in addition to the alternative decision variables using maps and graphs. The user provides the feedback via the Likert scale-based user rating and then this user rating is used by the micro-genetic algorithm operators to create the next generation of new alternatives (or, new chromosomes in the case of Genetic Algorithm). Hence, HS sessions are always presented successively and are equal to the number of generations of the micro-genetic algorithm. For example, in the progress of the illustrative experiment shown in Figure 2, since a micro-genetic algorithm with six generations was used, six HS sessions can be seen between the various introspection sessions. The automated search session (as seen in Figure 2 between introspection sessions 4 and 5) is the third type of session, which is a more computationally intensive optimization run and is performed by replacing the human user with a heuristic model of user ratings (or, a simulated decision maker model). The main purpose of automated search is to minimize user fatigue by replacing the human user with the simulated user, and hence no visual interfaces are shown to the user when automated search is running. Data on user ratings collected in earlier introspection and HS sessions are generally used to create the personalized and heuristic simulated decision maker models for every user. For example, Babbar-Sebens and Minsker (2012) used fuzzy logic models that related design parameters to user ratings, whereas in WRESTORE we have included multiple linear and non-linear classification models, neural networks, fuzzy logic models, and deep learning models (Singh, 2013) to create simulated decision maker models. 
In IGAMII, the sequence of interaction sessions (such as in Figure 2) is decided via a 448 flexible mixed initiative interaction (Hearst, 1999) strategy that monitors the individual user 449 learning and simulated decision maker model's accuracy to identify when human-guided search 450 should be conducted and when automated search should be conducted. Monitoring and tracking 451 user learning is an active topic of research in Human-Computer Interaction and Cognitive 452 Psychology. While additional research investigations will enable advanced tracking techniques 453 to inform the mixed initiative interaction strategies, WRESTORE currently uses the technique 454 proposed by Babbar-Sebens and Minsker (2012). This technique monitors the trends in users' 455 self-reported confidence in their user ratings to identify how fast human users are learning by 456 interacting with the tool. In this manner, it is possible to use the human user and the simulated 457 user models for search/optimization when they are most suitable for evaluation of alternatives. 458 After every optimization run, irrespective of whether it is human-guided search or automated 459 search, an introspection session is invoked to facilitate a user's re-reflection of previously 460 generated alternatives and improve her/his own cognitive learning.

461

462 2.3. WRESTORE Architecture: Figure 3 is a schematic configuration of the various 463 software and hardware components used to support the web-based WRESTORE tool. The 464 architecture model in WRESTORE is based on services provided by multiple servers (Garlan 465 and Shaw, 1993). The remote client users run their browser interfaces to access the various 466 services provided by the WRESTORE project website (http://wrestore.iupui.edu) that resides on 467 the Web Server. The web server interacts with the Database Servers and the main WRESTORE 468 Program Server to access additional services on storing, communicating, and processing user 469 data and instructions. 


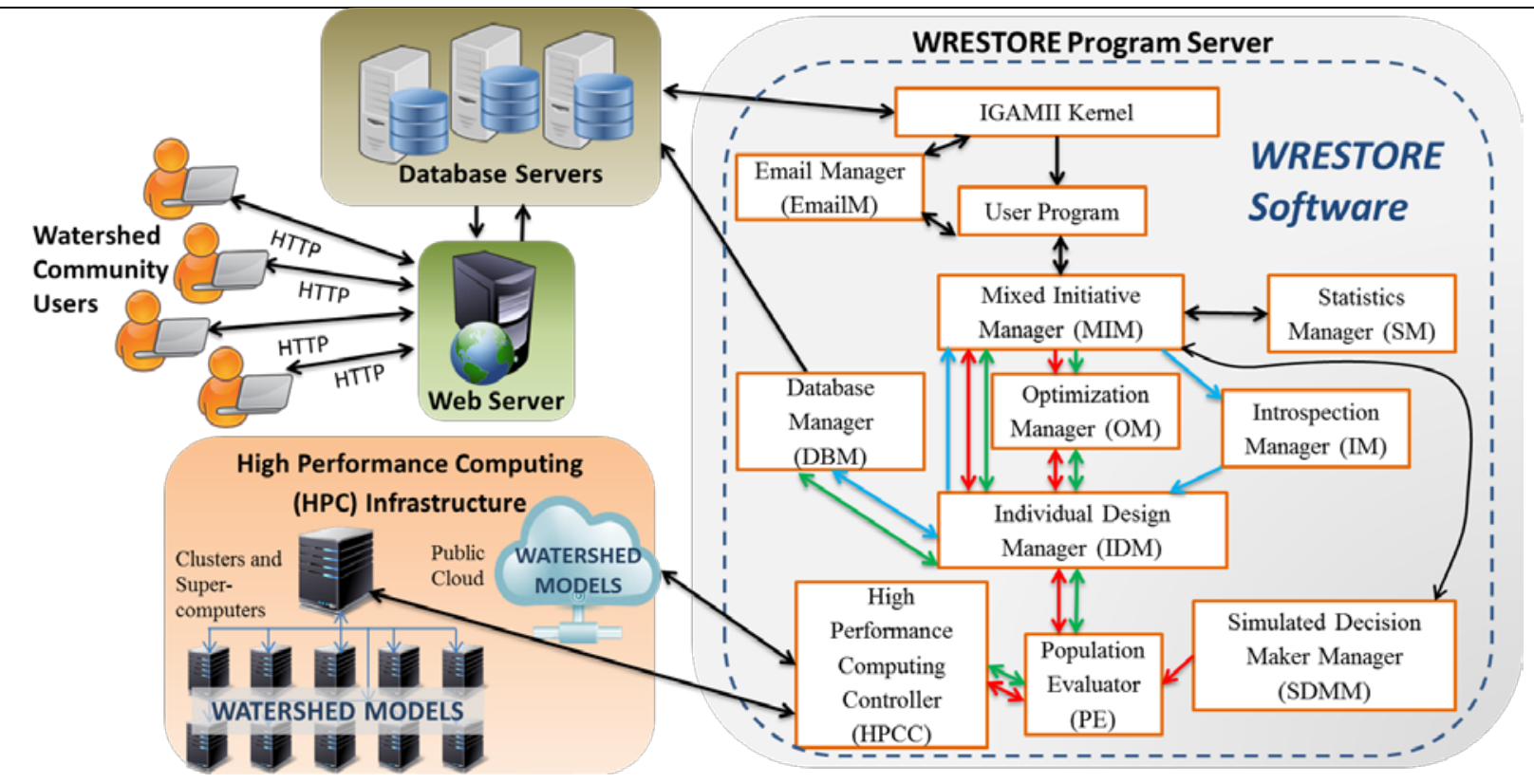

Figure 3. WRESTORE Architecture (Arrows indicate data flow. Blue arrows are executed specifically during introspection session, green arrows are executed specifically during human-guided search sessions, red arrows are executed specifically during automated search sessions)

471

472

473

474

475

476

477

478

479

Below is a description of the software services supported by the various server components in Figure 3.

(1) Web Server components: The Web Server hosts the project website with static and dynamic components developed using a combination of JavaScript, HTML, CSS, and PHP. The static components of the website are primarily informational and provide information on the tool and the watershed application to the users. Multiple Google Maps Image APIs have been included in the development of user friendly visualization of spatial data. The dynamic components of the website enable the users to create their own user accounts, and have real time access to the multiple services for starting and running instances of their own participatory search/optimization experiments.

(2) Database Server components: The Database Server runs MySQL for managing multiple databases that store data for users that have accounts on the website. This includes data related to user profiles and data specific to an actual real-time WRESTORE experiment run by the user. Every time a user initiates a search experiment in WRESTORE, the databases are accessed and updated by both the Web Server (via front end interfaces) and by the underlying main WRESTORE Program Server for processing. In this manner, all users 
have access to all alternatives found in the multiple experiments conducted by them over time.

(3) WRESTORE Program Server components: This is the main application program (written in Java) that runs the IGAMII-based participatory optimization methodology discussed earlier in Section 2.2. Below is a brief discussion on the various software components (or software managers) that coordinate specific tasks to accomplish the overall search methodology.

i. IGAMII Kernel: This is the main program that starts or stops instances of real-time search experiments for multiple authorized users who have previously registered on the project website.

ii. User Program: Every time a new experiment is started by the IGAMII Kernel, a new user program is initiated that associates a registered user with the new experiment, allocates database and computing resources to this specific user, and initializes various IGAMII parameters and other related software components (i.e. MIM, SM, OM, IM, IDM, SDMM, PE, HPCC, DBM, and VM listed and explained below) for the user. Similarly, when the experiment is completed, the user program de-allocates resources assigned to this user.

iii. Email Manager (EmailM): This is initiated by the IGAMII Kernel and handles the emailing system of the WRESTORE tool, for notifying users every time session data are available for viewing on the web interface. In this manner, users don't have to be continuously interacting in an ongoing experiment and can login to their account at a later convenient time to complete the rating of session alternatives.

iv. Mixed Initiative Manager (MIM): This component manages the mixed initiative interaction strategy of the IGAMII algorithm that was discussed earlier in Section 2.2.

v. Statistics Manager (SM): This conducts all the statistical tests (e.g. Mann Kendall tests on confidence data) to support the statistical analyses in mixed initiative interaction strategy in MIM.

vi. Optimization Manager (OM): Manages different types of underlying optimization algorithms used in human-guided search and automated search sessions. The default algorithm currently used for search is based on the Nondominated Sorting Genetic Algorithm (NSGA 2, Deb et al., 2002). 
vii. Introspection Manager (IM): Manages the multiple introspection sessions in which previously found alternatives that reside in the case-based memory table of the database are selected to be shown again to the user.

viii. Individual Design Manager (IDM): This works as an intermediary to communicate each alternative and its data to the other managers for processing and viewing, during every session.

ix. Simulated Decision Maker Manager (SDMM): Trains and tests different simulated decision maker models to predict a human's user ratings. These models are based on different Machine Learning algorithms. The best Machine Learning model is then chosen to perform automated search on behalf of the human.

x. Population Evaluator (PE): This manager receives alternatives from IDM, every time the alternatives need to be evaluated for their quantitative objectives (e.g., economic costs, peak flow reductions, etc.). These objectives are evaluated using mathematical objective functions that might require the use of process simulation models. For example, in the current WRESTORE we use the Soil and Water Assessment Tool (SWAT; Neitsch et al., 2005) watershed model to evaluate impact of conservation practices alternatives (as discussed in Section 2.1). However, the framework is flexible for incorporating other simulation models in future applications, if required. In order to run the simulation models for each of the alternatives, the PE sends them to the High Performance Computing Controller (HPCC) that interacts with high performance computing resources available to WRESTORE for running instances of the simulation models. When automated search is going on, the PE also interacts with the SDMM to obtain the best machine learning model for evaluating the user ratings of the alternatives.

xi. High Performance Computing Controller (HPCC): This manager connects the WRESTORE program server to available high performance computing infrastructure so that simulation models runtime can be reduced and users do not have a long waiting time. Multiple supercomputer, clusters and public cloud infrastructures can be accessed via the HPCC, based on available computing resources. In the past experiments with users, high performance Windows Tempest cluster at Indiana University, a dedicated ESA Windows cluster (Dell PowerEdge R620 servers with 112 nodes) at Oregon State 
University, and Amazon Cloud (http://aws.amazon.com/) have all been successfully used and tested.

xii. DB Manager (DBM): This manager collects all the processed data from the IDM and returns them to the Database servers so that they can then be sent to the web servers for visualization. It manages all the database connections and keeps track of their usage. Apart from traditional JDBC connection, Hibernate has also been implemented to operate the POJO (Plain Old Java Object) feature of Java in DBM.

2.3. WRESTORE Workflow and Interfaces: The arrows in Figure 3 indicate how the various components of the WRESTORE system work when a user initiates a search experiment. The entire system is based on JAVA RMI in asynchronous mode; hence, data are transferred from one component to another in an asynchronous manner. This allows multiple users to login at the same time and run their participatory search experiments independent of each other. For every user, the following workflow steps are currently performed:

(1) Based on what practices (related to decision variables discussed in Section 2.1) a user wants to explore in her/his watershed or sub-basin, and based on what goals (i.e. measures of performance discussed in Section 2.1) are important for the user, the user logs into the website and selects options on the BMPs and goals via the interface in Figure 4.

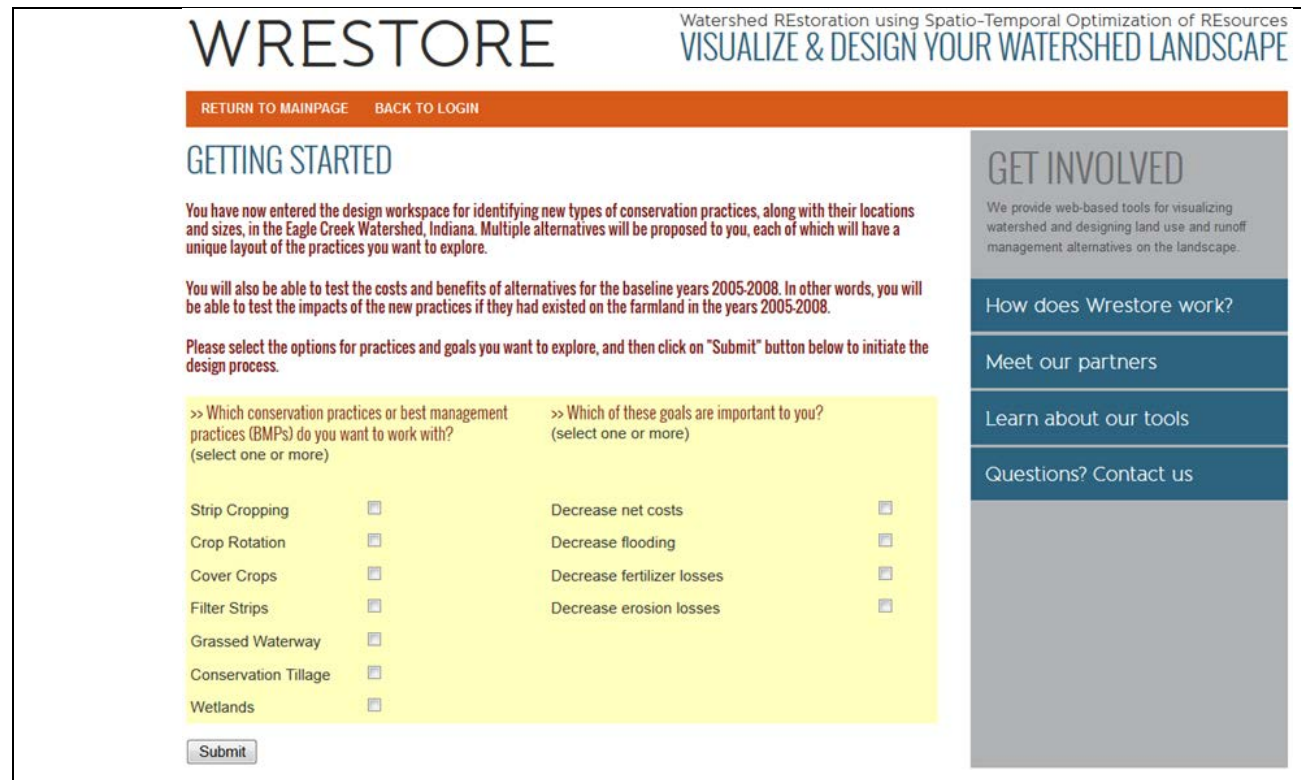

Figure 4. Interface for starting a new search experiment for the user's watershed of interest. 
(2) When the user submits her/his options, the Web Server passes that information to the database server (black arrows in Figure 3), which further sends a trigger notification to the IGAMII Kernel in WRESTORE Program Server. The IGAMII Kernel will initiate a search for every user; hence, multiple instances of the User Program in Figure 3 could be initiated at any point in time based on how many users are using the system. The managers EmailM, MIM, DBM, IDM, and HPCC are initialized. Once initiated, MIM initializes the remaining Managers - IM, OM, SDMM, SM, and PE - and then starts the IGAMII search experiment for the user.

(3) When a new User Program is initiated, the user will go through multiple interaction sessions, such as the ones shown in the progress bar in Figure 2. The search experiment in IGAMII, however, always first begins with an introspection session (i.e. Introspection 1 in Figure 2).

(4) In the first introspection session, the MIM will access the case-based memory (located in the database) to select potential watershed-scale alternatives found earlier in a different search or by an offline optimization run that did not involve any user ratings (e.g. a preliminary non-interactive optimization run proposed by Babbar-Sebens and Minsker 2012). The MIM then calls the IM, which sends these alternatives to the web server (via the IDM, DBM, and the database server) to show the alternatives to the user by means of a web-based interface (Figure 5). This same interface is also currently used for all humanguided search sessions, and is being further improved for better engagement with users. The User Program will then trigger the EmailM to send an email to the user whenever a session is available for viewing on the web server.

After the user logs into the website, she/he is able to visualize and compare the previously evaluated alternatives, which have now been made available to her/him for viewing in the first introspection session. The user evaluates all the alternatives shown by the interface based on her/his assessment of how BMPs are sited and sized in the entire watershed and in their local sub-basins of interest (viewed in the map space). The bar graphs on how alternatives perform with respect to quantitative goals (e.g., economic costs, etc.) allow the user to also evaluate them based on the performance of the alternatives in the entire watershed or in their local sub-basins of interest. The user provides feedback on her/his 

assessment of the quality of the alternative via user ratings, and these data along with typical interface usability data, are collected and sent back from the web server to the database for archiving and use by WRESTORE's software managers.

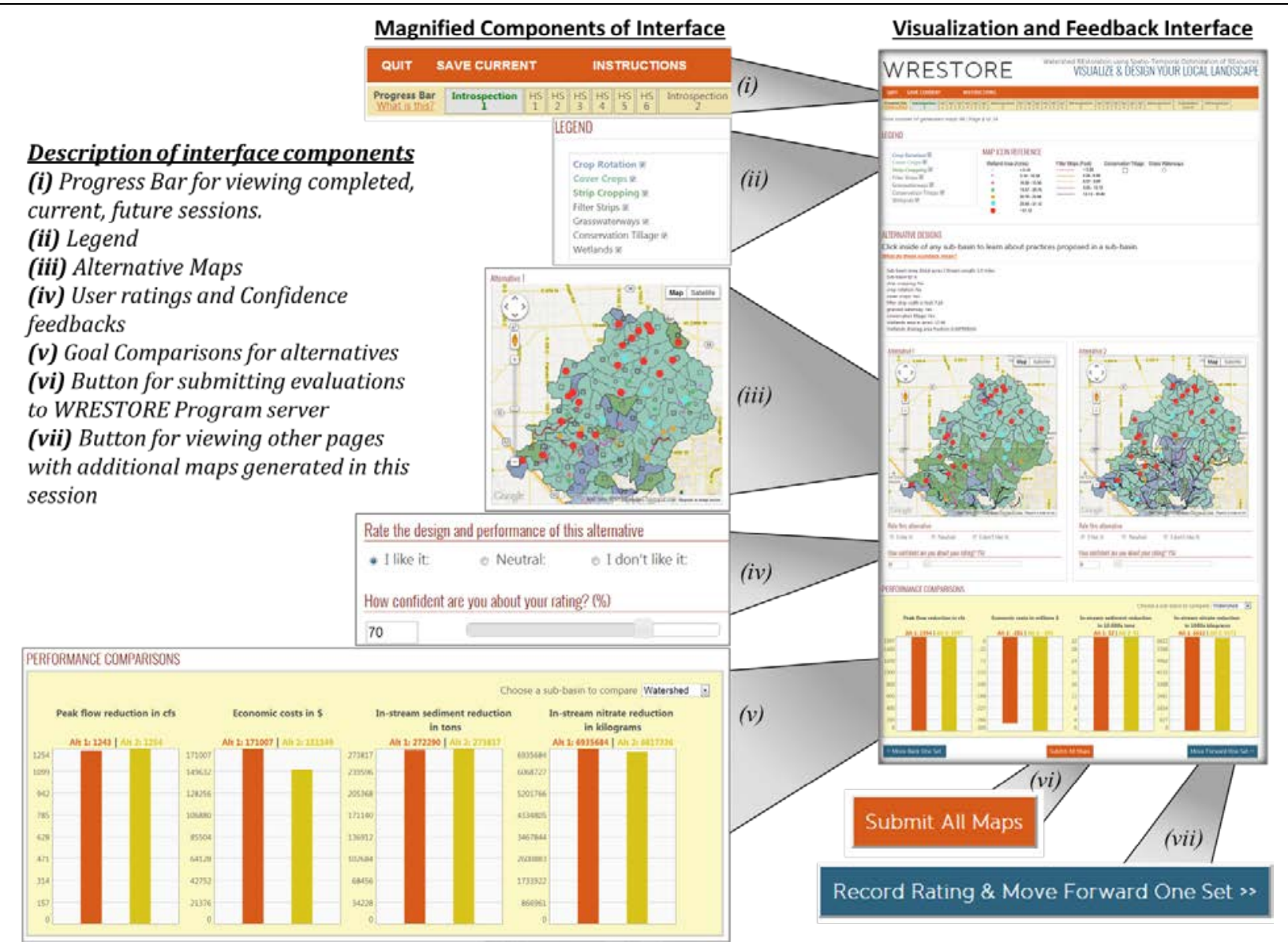

Figure 5. Visualization and Feedback Interface in WRESTORE

602

603

604

605

606

607

608

609

610

611

(5) After the introspection session is over, the MIM calls the SM to calculate multiple statistics on the usability data and for the mixed initiative interaction strategy. The MIM then invokes a call to OM to begin one of the two types of search sessions. For both HS and automated types of search sessions, the underlying optimization algorithm is initialized in a manner similar to that proposed and tested by Babbar-Sebens and Minsker (2012). For example, if NSGA2 is used, then $20 \%$ of the starting population is selected from the user's case-based memory and $80 \%$ are randomly created. Additionally, if MIM decides to start human-guided search, then the OM will use NSGA2 as a micro-GA with a small population size and few generations to minimize user fatigue. Whereas, if MIM decides to 
start automated search then the OM will use NSGA2 with larger population size and generations.

(6) The OM sends the alternatives proposed by underlying optimization algorithm's current iteration (or, generation in the case of NSGA2) to IDM, which communicates them to PE for numerical evaluation of the quantitative objective functions (or, performance goals as seen in bar graphs of Figure 5) and the user ratings.

a. To evaluate the quantitative objective functions, the PE will invoke the HPCC in order to run the process simulation models (i.e. watershed model of the application site) with different conservation practices (described in Section 2.1) activated in the sub-basins, as specified by the alternatives. Since this simulation of each alternative could take multiple minutes to run, the HPCC runs a job scheduler to efficiently distribute the simulation jobs to different computing nodes in real-time. If computing nodes are not free, then the simulation jobs for that user will be put in the waiting queue. Once the simulations are over, the HPCC returns the simulation results back to the PE for calculating necessary objective function values from the output files of the simulation models (as explained in Section 2.1).

b. If automated search is currently going on, then PE will also call the SDMM to invoke a suitable machine learning model that mimics the user to provide estimates of user ratings.

(7) Once the PE has evaluated all the alternatives in one iteration (which is also the session), the data on evaluated quantitative objective functions are sent to IDM that updates the data on alternatives. If automated search is currently going on, then the IDM, instead of sending the alternative to DBM, will send the data back to OM to start the next iteration (or, generation). However, in case of introspection sessions and human guided search sessions the IDM will send the data on alternatives to DBM, which will send the alternatives to the Database Server. The Database Server will then send a triggering message to the Web Server. At this point in time, if the introspection and human-guided search sessions are going on, then the IDM will also trigger the User Program (via the MIM) to send a notification email to user via the EmailM.

(8) For introspection sessions and human guided search sessions, the Web Server receives the trigger message for new incoming data, and then displays this new data on the alternatives 
into the visualization interface (Figure 5). The user provides her/his feedback, and the Web Server then informs the availability of the user feedback data to the DBM, which passes the data back to IDM. Once IDM receives the new data, if the user had just finished an HS session, the data are then sent to the OM to start the next iteration of HS session (or, human-guided optimization iteration). However, if an introspection session just finished, then a message is sent to MIM to initiate a new set of HS sessions. For both human-guided search and automated search if the maximum number or iterations (or, sessions) have not been completed, then the steps (6)-(8) will be repeated for each of the iterations of the underlying optimization algorithm. Once the HS sessions/iterations (e.g., HS1 to HS6 in Figure 2) are completed, the MIM will use the SM and SDMM to update the statistics and the simulated decision maker models. When either all of human-guided search sessions or automated search session end, the program moves to an introspection session in step (9).

(9) In this step, an introspection session will be initiated by the MIM (e.g., Introspection sessions 2, 3, 4, and 5 seen in Figure 2). The MIM will access the case-based memory (located in database) to select alternatives found earlier by the recent human-guided or automated searches. The IM is called, which sends these selected alternatives to the Web Server (via the IDM, DBM, and database servers) to show the alternatives to the user via the interface (Figure 5). The User Program will trigger the EmailM to send an email to the user whenever this session is available for viewing on the web server. Once the user has viewed and submitted her/his feedback, the data will move back to the database servers from the web server, and step (5) will be invoked again until the last introspection session, as specified in experiment settings, has been reached.

\subsection{WRESTORE Deployment for Multiuser Collaborative Design: Implementing} WRESTORE in a watershed involves three phases: pre-processing, real-time participatory design experiments, and post-processing. Currently, WRESTORE has been implemented, and tested for user learning, and multi-users engagement issues, and overall tool improvements at the test site of Eagle Creek Watershed, Indiana. But the flexible architecture of WRESTORE allows other watershed groups, in the future, to include their own simulation models, design parameters, and data related to their region. Figure 6 provides a synopsis of the three phases. 


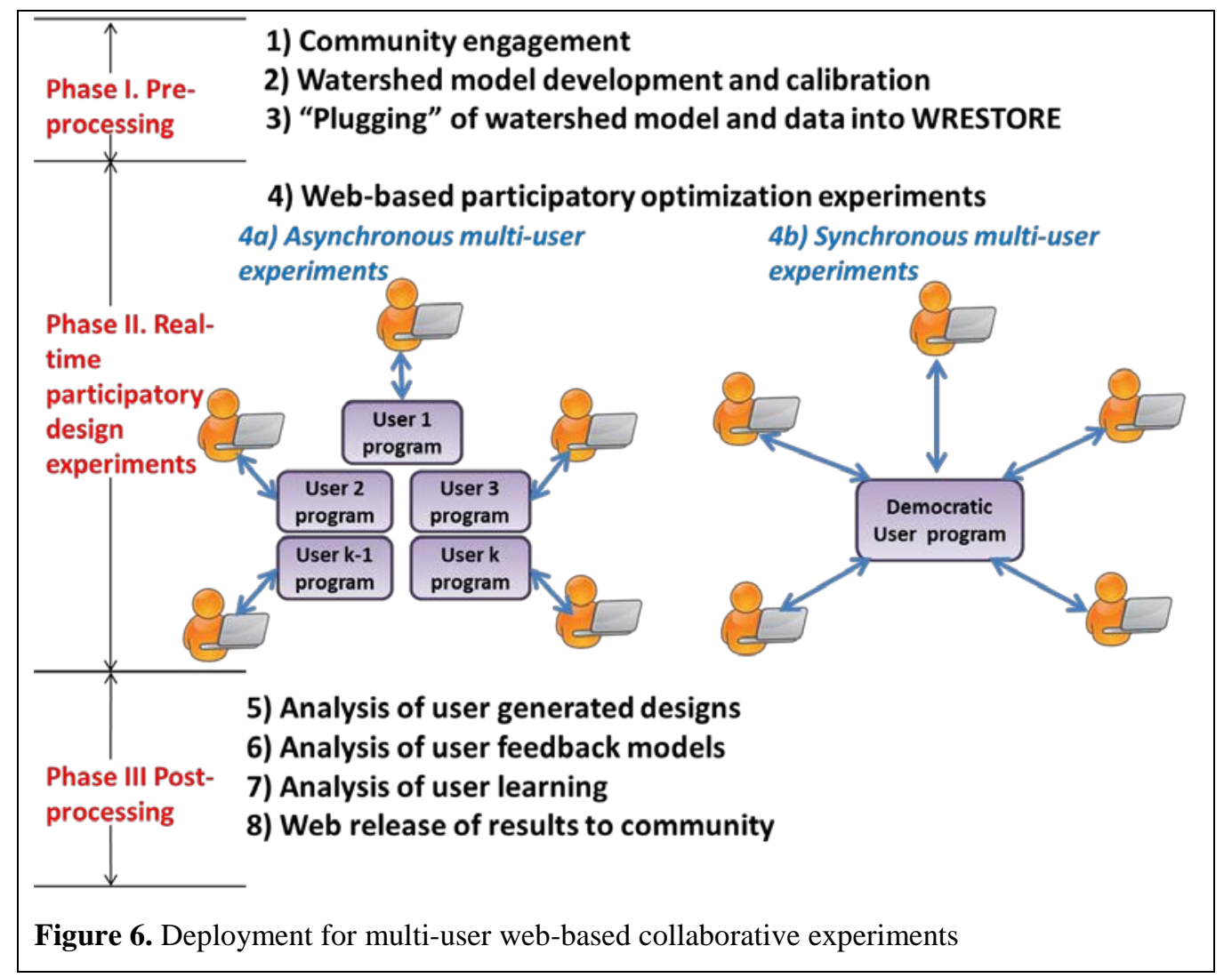

675 Phase I. Pre-processing phase: In this phase, a watershed community's agency personnel or 676 stakeholder council group/alliance is expected to first engage with the various parties of interest 677 to identify conservation practices of interest and specific sub-areas/sub-basins in their watershed 678 where potential sites for these practices could exist. While the nature of the engagement process 679 is beyond the scope of this article, it is expected that a shared vision of relevant goals and 680 constraints would be developed via this engagement process. The watershed community is 681 expected to then develop an appropriate process simulation model of their study area, preferably 682 via participatory modeling approaches (e.g. Palmer, 1998; Welp, 2001; Van Asselt Marjolein and 683 Rijkens-Klomp, 2002). We have currently used the SWAT model to simulate effectiveness of 684 new conservation practices in our test site, but WRESTORE's software architecture is not 685 constrained by a specific hydrology or water quality model. Once a simulation model has been 686 developed and calibrated, the watershed group leaders can then submit the model files to the 687 WRESTORE administrative team for setting up a WRESTORE project for their watershed. 688 Copies of the folders of the simulation model input/output/executable files are saved on the 689 WRESTORE program server, from where the program makes copies and saves them on to the 
HPC Infrastructure nodes whenever user experiments need to be conducted. Besides the simulation models, various GIS files identifying the watershed boundaries, sub-basins, and stream network are also required for the interface. These GIS data are stored into Google Fusion Tables so that Google Maps API can be used in the interface. We are currently in the process of developing a separate interface that will enable watershed group leaders to automate this setup process of site data and models for any watershed via the web.

Phase II. Real-time participatory design experiments: Once the WRESTORE project for the application watershed has been setup, it is then available for release to the general community. There are multiple approaches via which watershed groups could engage their stakeholders in conducting web-based, multi-user participatory optimization experiments in WRESTORE. Here, we present two of the approaches that have been tested.

i. Asynchronous multi-user experiments: In this type of experiment (see graphic (4a) in Figure 6), every user can initiate her/his own human-computer collaborative search for exploring spatial implementation of conservation practices that are of interest to her/him. Hence, multiple instances of User Program will be generated in this experiment type. When a user logs in and begins the WRESTORE workflow (discussed earlier in Section 2.4), she/he can choose from a set of available BMPs and goals for her/his watershed site. Multiple users can begin their experiments independent of others, and hence can asynchronously explore the effect of different types and combinations of conservation practices in the watershed. Since these experiments are conducted asynchronously (in a parallel fashion), WRESTORE currently does not assume a user's sub-basins of interest in advance, and, therefore, presumes that BMPs chosen (in the Figure 6 interface) by a user are applicable to all sub-basins in the watershed specified by the watershed group in Phase 1. Additionally, because of this assumption WRESTORE uses the values of the quantitative goals at the watershed scale (in the Figure 4 interface) as the objective functions for the underlying optimization algorithm. The future interface of WRESTORE will enable more detailed settings for individual users, where users will be able to declare a narrower sub-region of interest. The user-feedback-driven search and the learning process in the WRESTORE's underlying algorithms are, however, customized to individual participating users. One advantage of this kind of asynchronous engagement 
with multiple users is that it provides users the flexibility to explore alternatives at a time that suits them the most, without being dependent on the feedback of others.

ii. Synchronous multi-user experiments: In this type of experiment (see graphic (4b) in Figure 6), multiple users participate in a democratic human-computer collaborative search. A Democratic User Program is initiated that generates a set of alternatives that are shown to all users. Hence, synchronous participation is critical for this type of engagement setting so that the search process can advance once all feedbacks are obtained. Once all users have provided their user ratings, the majority user rating will be used as the final rating of the alternatives. The human-guided search, automated search and the learning process in WRESTORE's underlying algorithms are, therefore, customized to the majority opinion in the user community.

Phase III. Post-processing: Once user experiments are finished, alternatives generated by the multiple users can then be post-processed for similarities and dissimilarities in spatial plans of practices (i.e. alternatives) liked or disliked by the users. Additionally, simulated decision maker models generated by the WRESTORE program can be processed for identifying underlying parameters and variables that best explain the user ratings. Data collected via the interface on users can also be post-processed to understand how each participant engaged with the interface and whether any detectable learning or changes in opinions were observed. Once this postprocessing is completed, the analyses can be released to the user community for decision making and for identifying how individual user's behavioral factors affected identification of promising alternatives.

\section{SOFTWARE TESTS AND DISCUSSION}

The WRESTORE software is currently being tested for the study site of Eagle Creek Watershed, Indiana, (Figure 7) and with different types of users - i.e., university undergraduate and graduate students (from both Indiana University and Oregon State University), state agency personnel, and watershed stakeholders. While detailed research results with the different types of participants (including watershed stakeholders) will be provided in upcoming publications, here we provide results on software testing that used student users to demonstrate the benefits of the two types of real-time, web-based participatory optimization approaches discussed above. In the 
752 test plan, five student users (Participant IDs 2, 3, 4, 5, and 6) with background in Water 753 Resources were asked to do role-playing by assuming that they represented one of the colored 754 groups of sub-basins in Figure 7b and that they were interested in the suitability of BMPs only in 755 their local sub-basins group (e.g., Participant 2 was asked to focus on only red colored sub756 basins). The gray sub-basins in Figure 7a indicate all the sub-basins where new BMPs are being 757 considered for potential peak flow, nitrate reduction, and sediment reduction benefits. As

758 759 760 761 762

763 764 765 766 767 768 769 770 771 772 773 774 775 776 777 778 779 780 781 mentioned earlier, the SWAT model developed and calibrated for this watershed (Piemonti et al., 2013) was used to simulate baseline runoff and water quality conditions for the period of 20052008, and simulate effect of conservation practices on runoff and water quality for the same period.

For the test experiment, the participants were asked to consider cover crops and filter strips as potential BMPs for this watershed, and the alternatives for search experiments consisted of how these two practices were designed in the 108 gray sub-basins in Figure 7a. For cover crops, decisions were coded as binary variables, so when the practice was used in a specific sub-basin the variable had a value of 1 (and, 0 otherwise). For filter strips, the width of the strip was used as a decision variable and was allowed to vary from 0 to 5m. See Section 2.1 and Piemonti et al. (2013) for more details on how these decisions were encoded as practices into the SWAT model. The optimization algorithm used quantitative objective functions on maximizing peak flow reductions, minimizing costs, maximizing sediment reduction, and maximizing nitrate reductions, calculated at the watershed scale using the equations provided by Piemonti et al. (2013). To represent local subjective criteria, the participants were asked to provide user ratings (“I like it”, “Neutral”, and “I don’t like it”) for each alternative based on the design and performance of alternatives in their respective local areas. To help participants assess performance of practices in local areas, the same objective function equations in Piemonti et al. (2013) were also calculated for each local sub-basins. The participants, first, participated in the asynchronous user experiments, and then after five months participated in the synchronous user experiment. In each of these experiments, the five participants were made to go from Introspection 1 session to Introspection 4 session in Figure 2, with six human-guided search sessions between every two introspection sessions. In introspection 1, a set of alternatives found via a preliminary non-interactive optimization were shown to all the users so that they all had the 
783 same starting point for comparison purposes. This preliminary non-interactive optimization was 784 conducted using the NSGA 2 algorithm with the four quantitative objective functions. Since each 785 SWAT simulation model took about 10 minutes to run, with the HPC cluster (combination of 786 Tempest Cluster at Indiana University and ESA cluster in Oregon State University), the total 787 computational time for each of the experiments took about 180 minutes. Since every user had 788 individual variability on how much time they spent viewing and comparing alternatives on the 789 web-interface, the total clock time for the experiment was determined by the user's schedule and 790 varied from one to three days of engagement across users. 


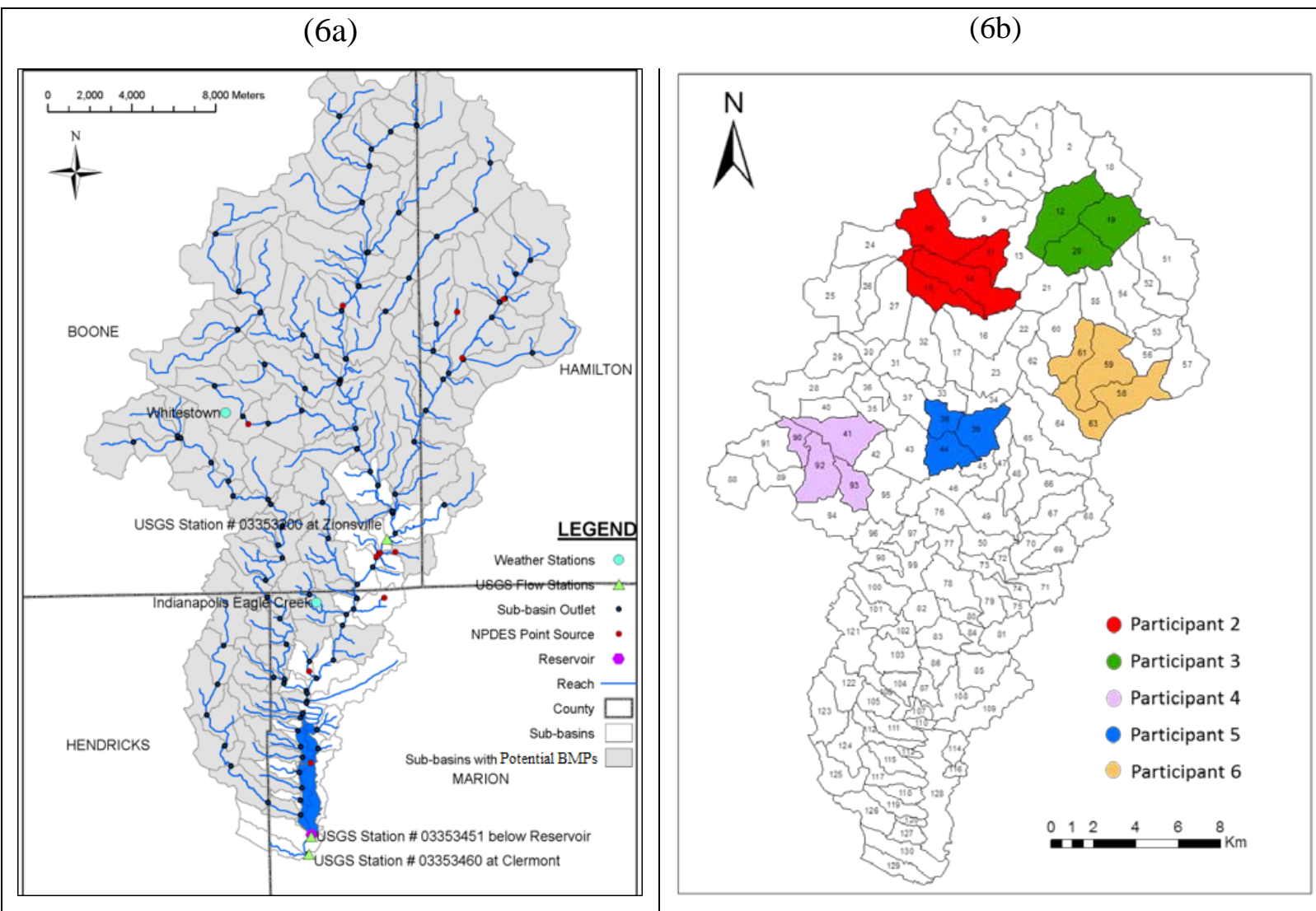

Figure 7. Eagle Creek Watershed sub-basins (6a) and sub-basins of interest to individual participants (6b)

794 The alternatives found by the participants in the two types of multi-user experiments were 795 compared with each other in objective space and in decision space. Figure 8 gives an overview 796 of the percent of alternatives with different user ratings that the participants found. It can be seen 797 that while for some participants (ID 2, 4, and 5) the percent of alternatives rated "I like it" 798 increased when the synchronous user experiment was performed, for others (participant IDs 3 799 and 6) the percent of "I Like it" alternatives actually decreased. Hence, either of the two engagement methods can be effective in helping users find alternatives that they like. The 801 democratic user's user rating was based on the majority rating of an alternative rated by the 802 individual participants. Hence, even though individually Participants 2, 4, and 5 found more "I 803 like it” alternatives, the overall democratic rating was affected by other participants and led to fewer percent of alternatives that were rated "I like it". 


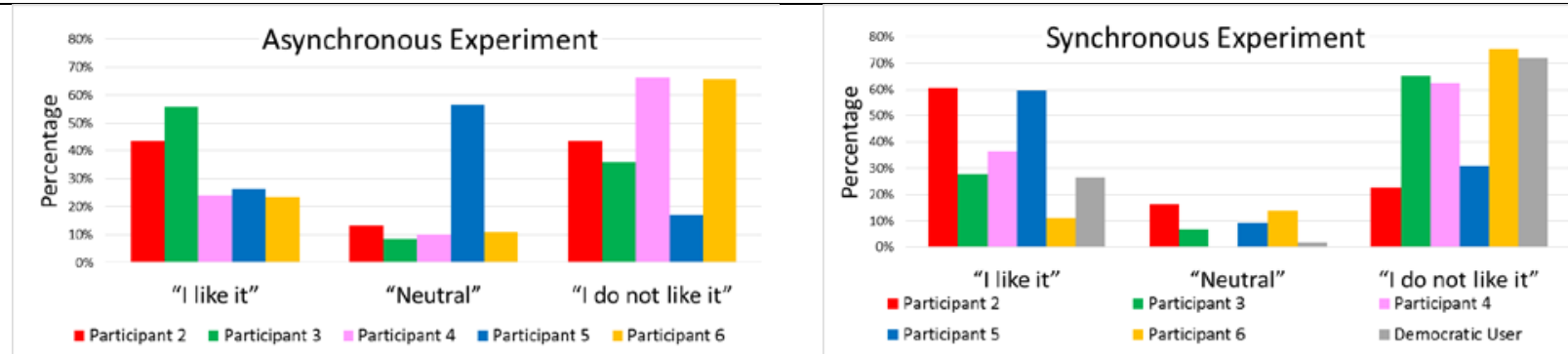

Figure 8. Percent of alternatives with the different user ratings in asynchronous and synchronous multi-user experiments

805

806

807

808

809

810

811

812

813

814

815

816

817

818

819

820

821

822

823

824

825

826
Figure 9 compares the post-processed alternatives in the quantitative objective function space (only peak flow reduction versus cost are shown), and further demonstrates the usefulness of WRESTORE. Figures (9a)-(9e) show the alternatives found by participants when they asynchronously conducted the user experiment, and Figure (9f) shows the democratic rating of the alternatives found during the synchronous collaborative experiment. Even for just these five users, multiple similarities and dissimilarities can be observed in the alternatives generated. For example, all participants agree that not all alternatives found by the non-interactive optimization (shown to them in Introspection 1) are above average or of user rating "I like it". In fact, Participants 4 and 5 found the majority of these non-interactive optimization alternatives to be of the type "I do not like it". Second, since WRESTORE customized the search to the user's feedback, different participants found "I like it" alternatives in different regions of the quantitative objective space, which did not necessarily coincide with the alternatives found by the non-interactive optimization. Participant 2 found a range of "I like it" alternatives that varied from high peak flow reductions with low costs to lower peak flow reduction with higher costs. Note that negative costs indicate economic revenue. Participant 3, 5, 6, and democratic user found their "I like it" alternatives in two visibly separated clustered regions. Participant 4 had a few number of alternatives in the region of lower peak flow reduction with higher costs. These results allow visualization of regions in quantitative objective function space where users might be willing to accept or reject alternatives. A typical non-interactive optimization that does not have the ability to include participant's preferences and perceptions via her/his user rating would typically reject many of these "I like it" alternatives. 

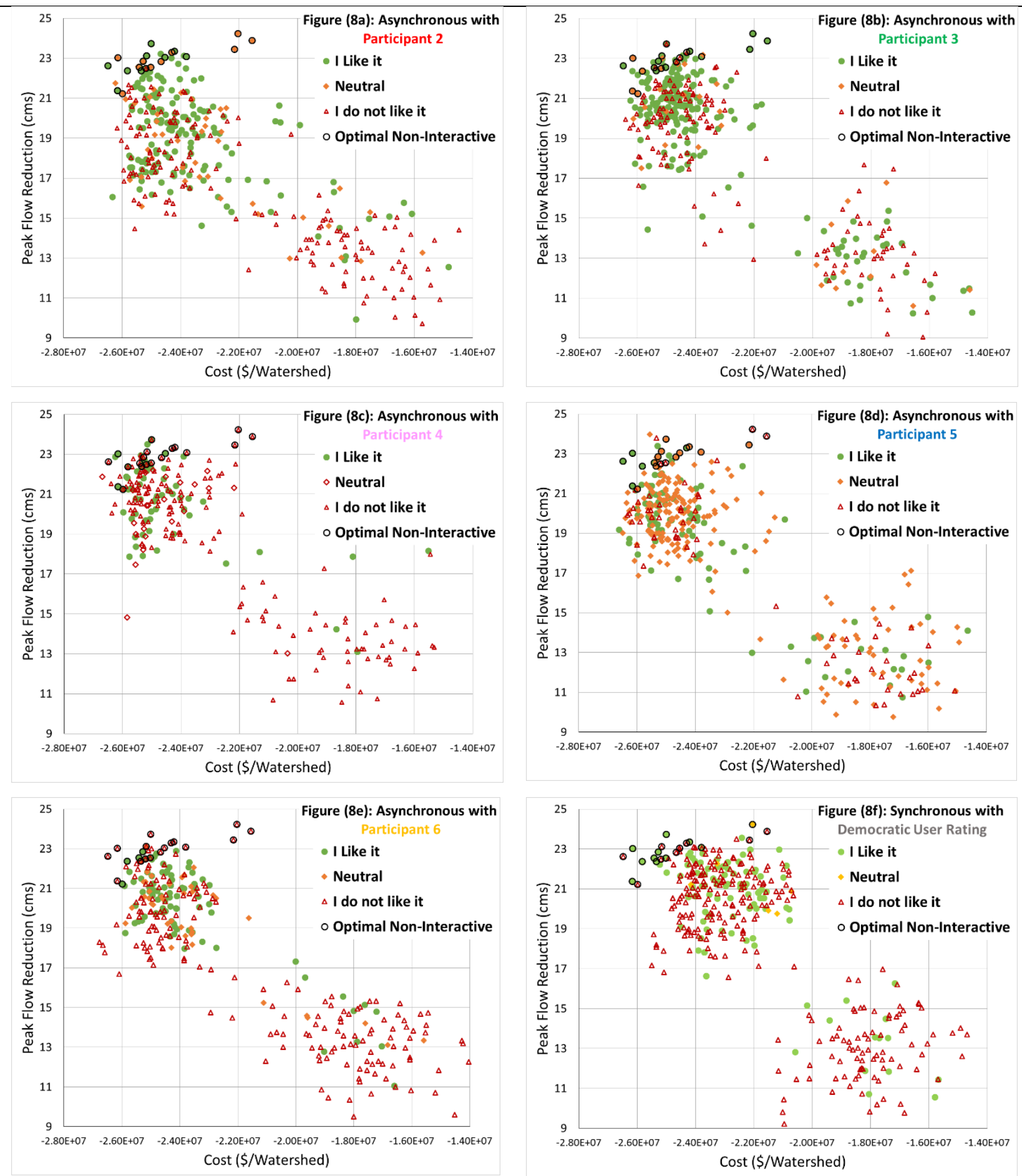

Figure 9. Alternatives with different user ratings found by participants and their performance in the quantitative objective function space.

828 Alternatives generated with the help of WRESTORE can be also be used to further identify 829 patterns in the decision space of the alternatives, and identify decisions that have higher chances 
of acceptability based on how the users perceived and rated them. Figure 10 shows statistics on the decision variables related to cover crops at the 108 candidate sub-basins (X axis) where new BMPs can be placed. Since, cover crops are coded as binary decisions in the search algorithm, all "I like it" rated alternatives found by every participant were sorted to find out the percent of alternatives that had cover crops (i.e. decision variable value $=1$ ) in the specific sub-basin. The $\mathrm{Y}$ axes in Figure 10 indicate this percent value as a probability. As visible from the two graphs in Figure 10, there is a large variability in the probability of cover crops in the 108 sub-basins (as seen by large scatter of probability values along $Y$ axis for every sub-basin), when the participants are allowed to conduct their own asynchronous search. When participants synchronously conduct the search using the democratic user rating procedure their overall disagreements in the probability of cover crops in the 108 sub-basins is reduced (as seen by a smaller scatter of probability values along $Y$ axis). The average variability (where, variabilitysubbasin $=$ maximum probabilitysub-basin-minimum probabilitysub-basin) in the probability of cover crops proposed by the participants was calculated to be 0.31 for asynchronous experiment and 0.19 for synchronous experiment. This indicates that the democratic user rating is more effective in finding alternatives that preserve the majority opinions on the values of the decision variables.

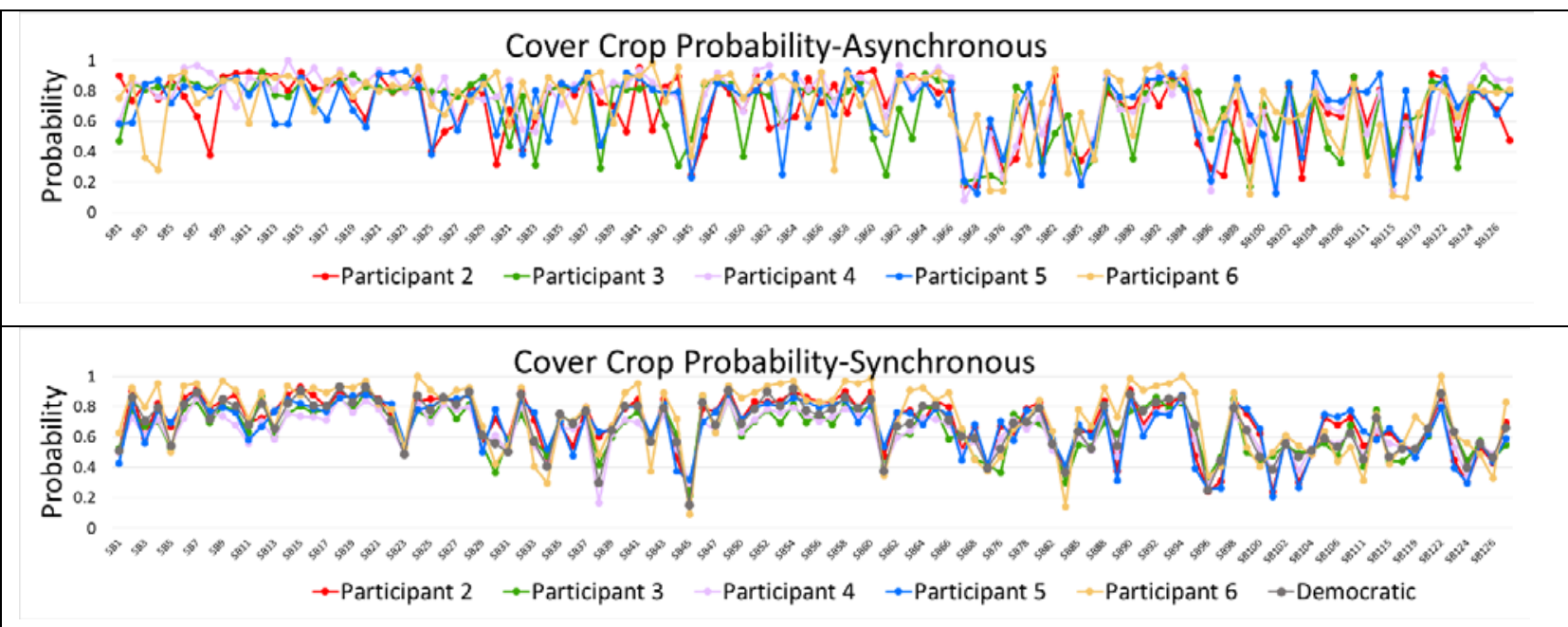

Figure 10. Probabilities of cover crops implemented in the various sub-basins of "I like it" alternatives

Figure 11 shows a similar trend in the statistics of the decision variables related to filter strips at the 108 candidate sub-basins ( $\mathrm{X}$ axis). For filter strips, the mode of the filter strip widths at each sub-basin was calculated, for all the "I like it" alternatives found by participants. The mode at 
851 every sub-basin represents the majority width value proposed by the "I like it" alternatives. The average disagreements in the mode values across all the sub-basins also decreased from 1.5 meters (for asynchronous experiment) to 0.85 meters (for synchronous experiment). This provides additional evidence in the benefit of conducting WRESTORE experiments in the synchronous mode, when increased agreement in the search of decision variable values is required.

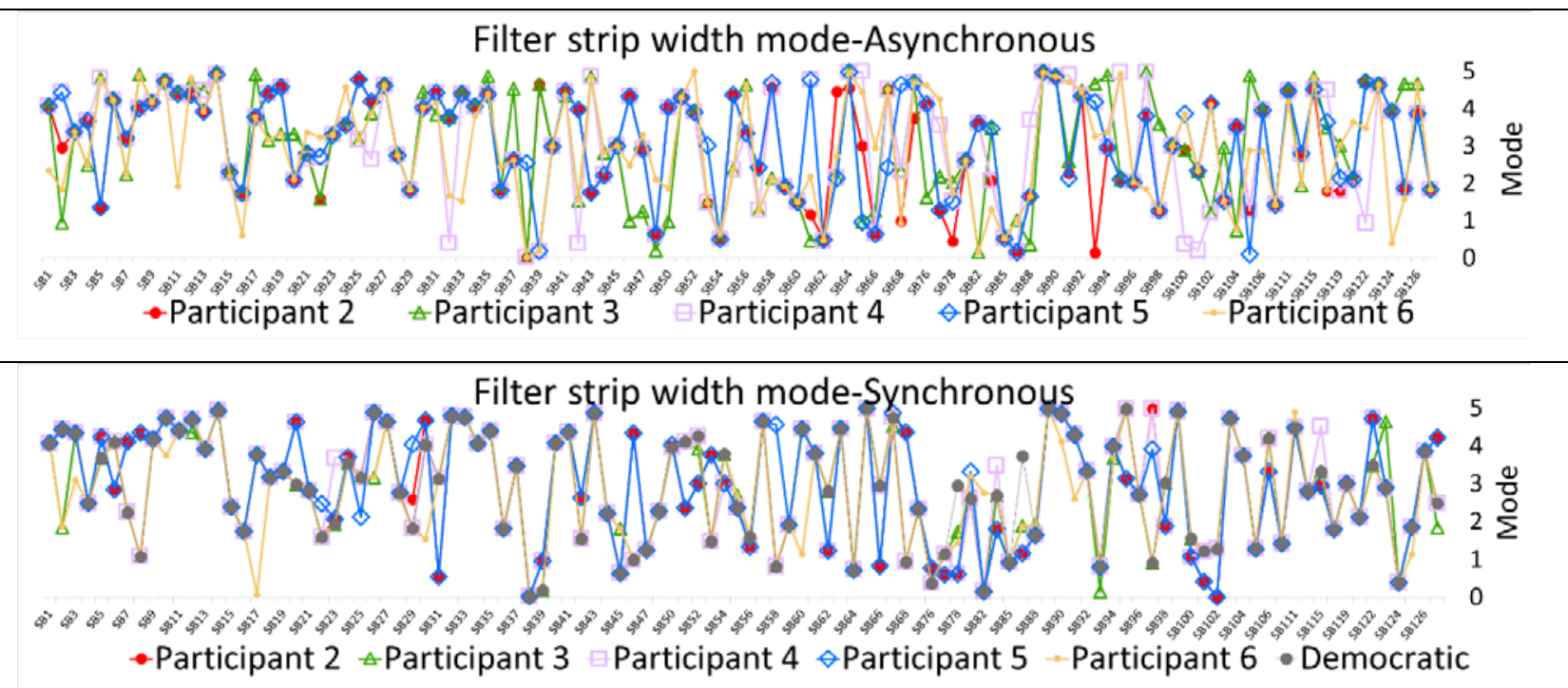

Figure 11. Mode of filter strip widths implemented in the various sub-basins of "I like it” alternatives

858

\section{CONCLUSIONS AND FUTURE DEVELOPMENTS}

With the ongoing advances in World Wide Web technologies and environments, use of online communities for collaboration and generation of solutions to real-world problems has become inevitable. The WRESTORE system provides an innovative and community-based approach for designing conservation practices on landscapes via web-based participation. Stakeholder groups and watershed planners have the potential to participate via the web to evaluate scenarios, optimize the scenarios, and generate customized alternatives that capture the communities' difficult-to-quantify criteria and concerns.

There are multiple strength and limitations of WRESTORE, which are being/will be addressed when future developments are released to the community: 
870 (i) While WRESTORE enables users to test the effectiveness of conservation practices using dynamic models, it assumes that such a model is readily available and the community has already gone through the model development and calibration phase. Additionally, the underlying code and architecture of WRESTORE is general enough to enable insertion of any other specific model that a watershed community might be interested in using, beyond the SWAT model that was used for the case study in this article. An interface for a community to select their specific simulation models and set up variables is currently being built and will be tested and demonstrated in future publications.

(ii) The implementation of WRESTORE is limited by the amount of time and computational resources taken by the embedded watershed model. Currently, the WRESTORE framework can be linked with the available research clusters and public Cloud to minimize time taken by simulation models; additional research for overcoming this barrier and decreasing user waiting time between sessions is also being conducted. For example, embedding faster surrogate models that can approximate watershed models is a potential solution to this problem.

(iii) For improving user engagement we are also conducting software usability tests and user studies with WRESTORE. These results will be used to include multiple improvements in future versions of the WRESTORE interfaces, including (a) a more game-like environment for users to directly modify alternatives at field scale and influence alternatives proposed by others, (b) enable users to compare alternatives with respect to climate change projections and other watershed impacts (e.g., impacts on habitat of indicator ecological species), and (c) enable watershed groups to create their own WRESTORE projects via the web-interface, etc.

(iv) One of the challenges in using such web-based design environments is the protection of privacy when users explore the alternatives. Since WRESTORE is a research tool at this point in time, all data shared by users are kept confidential and not shared with anyone else beyond the research team approved by the university's Institutional Review Board. Additionally when user data are utilized by the WRESTORE architecture, identifiers are removed from the data to maintain privacy of specific users. In future developments we plan to provide adaptive privacy settings to users to allow them to control the visibility of their participation. 


\section{ACKNOWLEDGEMENTS}

903

904

905

906

907

908

909

910

911

912

913

914

915

916

917

918

919

920

921

922

923

924

925

926

927

928

929

930

931

This study has been supported by National Sciences Foundation award ID \#1332385 (previously ID\# 1014693). We would also like to thank Ms. Jill Hoffmann of Empower Results LLC for her help with organizing multiple workshops with stakeholders and to enable testing of the tool, and Mr. Jon Eynon for the website and the initial interface development. We are grateful to the many students who have participated in the user tests or participated in conducting research and code developments on multiple aspects of this tool. Finally, we would like to acknowledge the effort of the reviewers whose feedbacks were valuable in helping us improve this manuscript.

\section{REFERENCES}

Andradóttir, S., 1998. Simulation optimization. In: Banks, J. (Ed.), Handbooks of Simulation: Principles, Methodology, Advances, Applications, and Practice. John Wiley \& Sons, New York (Chapter 9).

Arabi, M., Frankenberger, J.R., Engel, B.A., and Arnold, J.G., 2007. Representation of Agricultural Conservation Practices with SWAT. Hydrological Processes, 22(16), 3042-3055.

Argent, R.M., and Grayson, R.B., 2003. A modelling shell for participatory assessment and management of natural resources. Environmental Modelling \& Software, Vol. 18, pp. 541-551.

Arnold, J.G., Allen, P.M., and Morgan, D., 2001. Hydrologic Model for Design of Constructed Wetlands. Wetlands, 21(2), 167-178.

Arnold, J.G., Potter, K.N., King, K.W., and Allen, P.M., 2005. Estimation of Soil Cracking and the Effect on Surface Runoff in a Texas Blackland Prairie Watershed. Hydrological Processes, 19(3), 589-603. Assaf, H., van Beek, E., Borden, C., Gijsbers, P., Jolma, A., Kaden, S., Kaltofen, M., Labadie, J. W., Loucks, D. P., Quinn, N. W. T., Sieber, J., Sulis, A., Werick, W. J., and Wood, D. M., 2008. Generic Simulation Models for Facilitating Stakeholder Involvement in Water Resources 
932 Planning and Management: A Comparison, Evaluation, and Identification of Future Needs, 933 Chapter 13 of Environmental Modelling, Software and Decision Support: state of the art and new 934 perspectives, Jakeman, A.J., Voinov, A.A., Rizzoli, A.E. and Chen, S.H. (eds), Elsevier series on 935 Developments in Integrated Environmental Assessment. Elsevier Ltd.

936

937 Babbar-Sebens, M., and Minsker, B.S., 2012. Interactive Genetic Algorithm with Mixed 938 Initiative Interaction for multi-criteria ground water monitoring design. Appl. Soft 939 Comput.,12(1):182-95.

940

941 Babbar-Sebens, M., Barr, R.C., Tedesco, L.P., and Anderson, M., 2013. Spatial identification 942 and optimization of upland wetlands in agricultural watersheds. Ecological Engineering, Volume 943 52, Pages 130-142.

944

945 Beckley, T.M., Parkins, J.R., Sheppard, S.R.J., 2006. Public Participation in Sustainable Forest 946 Management: a Reference Guide. Sustainable Forest Management Network, Edmonton, Alberta. 94755 p.

948

949 Bierle, T.C., 1999. Using social goals to evaluate public participation. Policy Studies Review 16, $950 \quad 75-103$.

951

952 Bracmort, K. S., Arabi, M., Frankenberger, J. R., Engel, B. A. and Arnold, J. G., 2006. Modeling 953 long-term water quality impact of Structural BMPs. Trans. ASABE, 49(2), 367-374.

954

955 Brown, G.G., and Reed, P., 2009. Public participation GIS: a new method for use in national 956 forest. Forest Science 55(2):166-182.

957

958 Brown, G., and Weber, D., 2011. Public participation GIS: a new method for national park 959 planning. Landscape and Urban Research, 102(1):1-15.. 
961 Castelletti, A., and Soncini-Sessa, R., 2006. A procedural approach to strengthening integration 962 and participation in water resource planning. Environmental Modelling and Software 21, 14559631470.

964

965 Castelletti, A., and Soncini-Sessa, R., 2007. Coupling real-time control and socioeconomic issues 966 in participatory river basin planning. Environmental Modelling and Software 22, 1114-1128.

967

968 Chien, S., Rabideau, G., Willis, J., Mann, T., 1999. Automating planning and scheduling of 969 shuttle payload Operations. J. Artif. Intell., 114, pp. 239-255.

970

971 Conrad, C.C., Hilchey, K.G., 2011. A review of citizen science and community-based 972 environmental monitoring: issues and opportunities. Environmental Monitoring and Assessment 973 176, 273-291.

974

975 Craw, S., 2003. Introspective learning to build case-based reasoning (CBR) knowledge 976 container, Lecture Notes in Computer Science, 2734/ Springer, Berlin.

977

978 Daniels, S.E., Walker, G.B., 2001. Working Through Environmental Conflict: the

979 Collaborative Learning Approach, Praeger, Santa Barbara, CA, USA.

980

981 Deb, K., Pratap, A., Agarwal, S., and Meyarivan, T., 2002. A fast and elitist Multiobjective 982 Genetic Algorithm: NSGA-II. IEEE Trans. Evol. Comput. 6 (April (2)), 182-197.

983

984 Döll, C., Döll, P., and Botsb, P., 2013. Semi-quantitative actor-based modelling as a tool to 985 assess the drivers of change and physical variables in participatory integrated assessments. 986 Environmental Modelling \& Software, Vol. 46, pp. 21-32.

988 do Nascimento, H.A.D., and Eades, P., 2002. User hints for directed graph drawing. In: Proc. 989 Graph Drawing, pp. 205-219. Springer, Berlin. 
991 Esty, D.C., 2004. Environmental protection in the information age. New York University Law 992 Review 79.

Fisher, M.L., 1985. Interactive Optimization. Annals of Operations Research, 5(6), pp. 541-556.

995

Fu, M., 1994. Optimization via simulation: a review. Ann. Oper. Res. 53, 199-248.

Fu, M., 2002. Optimization for simulation: theory vs. practice. INFORMS J. Comput. 14 (3), 999 $192-215$.

Garlan, D. and Shaw, M., 1993. An Introduction to Software Architecture, Advances in Software Engineering and Knowledge Engineering, Vol. 1, World Scientific Publishing Co., Singapore.

Gosavi, A., 2003. Simulation-Based Optimization: Parametric Optimization Techniques and 1005 Reinforcement Learning. Kluwer Academic Publishers, Norwell, MA, USA.

Haimes, Y. and Hall, W., 1974. Multiobjectives in Water Resource Systems Analysis: the 1008 Surrogate Trade-off Method. Water Resources Research, 10:615-624.

Hamilton, S.H., ElSawah, S., Guillaume, J.H.A., Jakeman, A.J., Pierce, S.A. 2015. Integrated 1011 assessment and modelling: Overview and synthesis of salient dimensions, Environmental 1012 Modelling \& Software, Volume 64, February, Pages 215-229, ISSN 1364-8152, 1013 http://dx.doi.org/10.1016/j.envsoft.2014.12.005.

1015 Hearst, M.A., 1999. Trends and controversies: mixed-initiative interaction. IEEE Intelligent 1016 Systems 14 (5), 14-23.

1018 Hey, D.L., Montgomery, D.L., Urban, L., Prato, T., Fordes, A., Martell, M., Pollack, J., Steele, 1019 Y., and Zarwell, R., 2004. Flood Reduction in the Upper Mississippi River Basin: An Ecological 1020 Means, The Wetlands Initiative Report funded by the McKnight Foundation, 1021 (http://www.wetlands-initiative.org/Flood Damage.html). 
1023 Hudson-Smith, A., Batty, M., Crooks, A., Milton, R., 2009. Mapping for the masses: accessing 1024 web 2.0 through crowdsourcing. Social Science Computer Review 27, 524-538.

1025

1026 Kangas, J., Store, R., 2003. Internet and teledemocracy in participatory planning of natural 1027 resources management. Landscape and Urban Planning 62, 89-101.

1028

1029 Kearns, F.R., Kelly, M., Tuxen, K.A., 2003. Everything happens somewhere: using webGIS as a 1030 tool for sustainable natural resource management. Frontiers in Ecology and the Environment 1, $1031541-548$.

1032

1033 Kelly, M., Ferranto, S., Lei, S., Ueda, K., Huntsinger, L., 2012. Expanding the table: The web as 1034 a tool for participatory adaptive management in California forests. Journal of Environmental 1035 Management, 109: 1-11.

1036

1037

Klau, G.W., N. Lesh, J. Marks, M. Mitzenmacher. 2009. Human-guided search, J Heuristics, 1038 DOI 10.1007/s10732-009-9107-5.

1039

1040

Law, A., Kelton, W., 2000. Simulation Modeling and Analysis, Third Ed. McGraw-Hill, New 1041 York.

1042

1043

1044

1045

1046

1047

McCown, R.L. 2002. Changing systems for supporting farmers’ decisions: problems, paradigms, 1048 and prospects. Agricultural Systems, 74: 179-220.

1049

1050

McIntosh, B.S. , Ascough II, J.C., Twery, M., Chew, J., Elmahdi, A., Haase, D., Harou, J.J., 1051 Hepting, D., Cuddy, S., Jakeman, A.J., Chen, S., Kassahun, A., Lautenbach, S., Matthews, K., 1052 Merritt, W., Quinn, N.W.T., Rodriguez-Roda, I., Sieber, S., Stavenga, M., Sulis, A., Ticehurst, 
1053 J., Volk, M., Wrobel, M., van Delden, H., El-Sawah, S., Rizzoli, A., Voinov, A. 2011. 1054 Environmental decision support systems (EDSS) development - Challenges and best practices, 1055 Environmental Modelling \& Software, Volume 26, Issue 12, December, Pages 1389-1402, ISSN 1056 1364-8152, http://dx.doi.org/10.1016/j.envsoft.2011.09.009.

1057

1058 Mintzberg H, Raisinghani D, Théorêt A., 1976. The Structure of “Unstructured” Decision 1059 Processes. Adm Sci Q; 21(2):246-75.

1061 Neitsch, S.L., Arnold, J.G., Kiniry, J.R., Williams, J.R., 2005. Soil and Water Assessment Tool 1062 Theoretical Documentation - Version 2005. Grassland, Soil and Water Research Laboratory, 1063 Agricultural Research Service and Blackland Research Center, Texas Agricultural Experiment 1064 Station, Temple, TX.

1065

1066 O’Reilly, T., 2007.What is web 2.0: design patterns and business models for the next generation 1067 of software. Communications and Strategies 65, 17-37.

1068

Palmer, R.N., 1998. A history of shared vision modeling in the ACT-ACF comprehensive study: A modeler’s perspective. Coord Water Resour Environ, 221-6.

1071

Piemonti, A.D., M. Babbar-Sebens, E.J. Luzar, 2013. Optimizing conservation practices in 1073 watersheds: Do community preferences matter? Water Resources Research, Vol. 49, pp. 1-25, 1074 DOI: 10.1002/wrcr.20491.

1075

1076 Rabotyagov, S., Campbell, T., Valcu, A., Gassman, P., Jha, M., Schilling, K., Wolter, C., and 1077 Kling, C., 2013. Spatial multiobjective optimization of agricultural conservation practices using 1078 a SWAT model and an evolutionary algorithm. Journal of Visualized Experiments, Vol. 1079 70(e4009), 1-11.

1080

Rinner, C., Kefller, C., Andrulis, S., 2008. The use of Web 2.0 concepts to support deliberation 1082 in spatial decision-making. Computers, Environment and Urban Systems, 32, 386-395. 
1084 Selin, S.W., Pierskalla, C., Smaldone, D., Robinson, K., 2007. Social learning and building trust 1085 through a participatory design for natural resource planning. Journal of Forestry, 105, 421-425.

Sheppard, S.R.J., Meitner, M., 2005. Using multi-criteria analysis and visualization for 1088 sustainable forest management planning with stakeholder groups. Forest Ecology \& 1089 Management, 207, 171-187.

1090

Shi, Z., Zhang, S., 2005. Case-based introspective learning, in: Fourth IEEE Conference on 1092 Cognitive Informatics (ICCI 2005), August 8-10, pp. 43-48.

1093

Simon HA, 1977. The new science of management decision. Prentice-Hall.

1095

1096

Singh, V.B., 2013. User modeling and optimization for environmental planning system design. 1098 Masters thesis, Purdue University.

Soncini-Sessa, R., A. Castelletti, and E. Weber, 2007. Integrated and participatory water resources management: Theory. Elsevier, Amsterdam, NL.

Takagi, H. 2001. Interactive evolutionary computation: Fusion of the capabilities of EC optimization and human evaluation, in Proceedings of the IEEE, 89(9), 1275-1296.

Van Asselt Marjolein BA, Rijkens-Klomp N., 2002.A look in the mirror: Reflection on participation in Integrated Assessment from a methodological perspective. Glob. Environ. Chang.,12(3):167-84.

1114 Wilcove, D.S., 2004. The private side of conservation. Front Ecol Environ, 2, 326-331. 
1116 Welp M., 2001. The use of decision support tools in participatory river basin management. Phys

1117 Chem Earth, Part B Hydrol Ocean Atmos, 26(7-8):535-9.

1118

1119 Yates, D., D. Purkey, J. Sieber, A. Huber-Lee, and H. Galbraith, 2005a. WEAP21—A demand-, 1120 priority-, and preference-driven water planning model. part 2: Aiding freshwater ecosystem 1121 service evaluation. Water Int., 30, $501-512$.

1122

1123 Yates, D., J. Sieber, D. Purkey, and A. Huber-Lee, 2005b. WEAP21-A demand-, priority-, and 1124 preference-driven water planning model. part 1. Water Int., 30, 487 - 500. 